\title{
RESEARCH
}

Open Access

\section{The FAM3C locus that encodes interleukin- like EMT inducer (ILEI) is frequently co- amplified in MET-amplified cancers and contributes to invasiveness}

Ulrike Schmidt ${ }^{1}$, Gerwin Heller ${ }^{2}$, Gerald Timelthaler ${ }^{3}$, Petra Heffeter $^{3}$, Zsolt Somodi ${ }^{4,5}$, Norbert Schweifer ${ }^{6}$, Maria Sibilia ${ }^{3}$, Walter Berger ${ }^{3}$ and Agnes Csiszar ${ }^{3^{*}}$

\begin{abstract}
Background: Gene amplification of MET, which encodes for the receptor tyrosine kinase c-MET, occurs in a variety of human cancers. High c-MET levels often correlate with poor cancer prognosis. Interleukin-like EMT inducer (ILEI) is also overexpressed in many cancers and is associated with metastasis and poor survival. The gene for ILEI, FAM3C, is located close to MET on chromosome 7q31 in an amplification "hotspot", but it is unclear whether FAMC3 amplification contributes to elevated ILEI expression in cancer. In this study we have investigated FAMC3 copy number gain in different cancers and its potential connection to MET amplifications.

Methods: FAMC3 and MET copy numbers were investigated in various cancer samples and 200 cancer cell lines. Copy numbers of the two genes were correlated with mRNA levels, with relapse-free survival in lung cancer patient samples as well as with clinicopathological parameters in primary samples from 49 advanced stage colorectal cancer patients. ILEI knock-down and c-MET inhibition effects on proliferation and invasiveness of five cancer cell lines and growth of xenograft tumors in mice were then investigated.

Results: FAMC3 was amplified in strict association with MET amplification in several human cancers and cancer cell lines. Increased FAM $3 C$ and MET copy numbers were tightly linked and correlated with increased gene expression and poor survival in human lung cancer and with extramural invasion in colorectal carcinoma. Stable ILEI shRNA knock-down did not influence proliferation or sensitivity towards C-MET-inhibitor induced proliferation arrest in cancer cells, but impaired both c-MET-independent and -dependent cancer cell invasion. c-MET inhibition reduced ILEI secretion, and shRNA mediated ILEl knock-down prevented c-MET-signaling induced elevated expression and secretion of matrix metalloproteinase (MMP)-2 and MMP-9. Combination of ILEl knock-down and c-MET-inhibition significantly reduced the invasive outgrowth of $\mathrm{NCl}-\mathrm{H} 441$ and $\mathrm{NCl}-\mathrm{H} 1993$ lung tumor xenografts by inhibiting proliferation, MMP expression and E-cadherin membrane localization.

(Continued on next page)
\end{abstract}

\footnotetext{
* Correspondence: agnes.csiszar@meduniwien.ac.at

${ }^{3}$ Department of Medicine I, Institute of Cancer Research, Medical University of Vienna, Borschkegasse 8a, A-1090 Vienna, Austria

Full list of author information is available at the end of the article
}

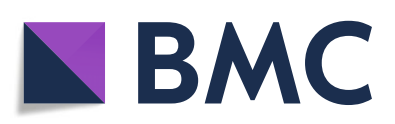

(- The Author(s). 2021 Open Access This article is licensed under a Creative Commons Attribution 4.0 International License, which permits use, sharing, adaptation, distribution and reproduction in any medium or format, as long as you give appropriate credit to the original author(s) and the source, provide a link to the Creative Commons licence, and indicate if changes were made. The images or other third party material in this article are included in the article's Creative Commons licence, unless indicated otherwise in a credit line to the material. If material is not included in the article's Creative Commons licence and your intended use is not permitted by statutory regulation or exceeds the permitted use, you will need to obtain permission directly from the copyright holder. To view a copy of this licence, visit http://creativecommons.org/licenses/by/4.0/ The Creative Commons Public Domain Dedication waiver (http://creativecommons.org/publicdomain/zero/1.0/) applies to the data made available in this article, unless otherwise stated in a credit line to the data. 
(Continued from previous page)

Conclusions: These novel findings suggest MET amplifications are often in reality MET-FAM3C co-amplifications with tight functional cooperation. Therefore, the clinical relevance of this frequent cancer amplification hotspot, so far dedicated purely to c-MET function, should be re-evaluated to include ILEI as a target in the therapy of c-METamplified human carcinomas.

Keywords: Interleukin-like EMT inducer (ILEI), FAM3C, C-MET, Gene amplification, Invasion, Matrix metalloproteinase $(\mathrm{MMP})$, Cancer

\section{Background}

Cancer develops via a complex multistep process [1]. Central to this are multiple genetic abnormalities that lead to disruption of the normal cell signaling networks. An example is when proto-oncogenes with physiological functions within healthy cells become hyperactive or dysregulated leading to uncontrolled cell growth. This can be the result of a mutation that switches an oncogene on permanently or from overexpression of the protein [2].

$M E T$ is a proto-oncogene that encodes a receptor tyrosine kinase (RTK), c-MET, a receptor for hepatocyte growth factor (HGF). c-MET is expressed in many epithelial cells, while HGF is secreted from mesenchymal cells. Their interaction stimulates various signaling pathways and has a vital role in breaking cell adhesions to promote motility of epithelial cells during embryogenesis and wound healing [3]. However, hyperactivity of cMET is evident in a wide range of cancers where it can drive proliferation, survival, motility, and invasion [4]. In particular, high levels of c-MET often correlate with poor prognosis in cancer patients [4]. In some cancers, such as gastric and non-small cell lung carcinomas (NSCLCs), hyperactivity of c-MET is the result of multiple copies of the $M E T$ gene and these cells seem to be largely dependent on sustained c-MET activity for their growth and survival [3].

An increased gene copy number $(\mathrm{CN})$ can result from gene amplification or aneuploidy. Gene amplification "hotspots" are mapped throughout the human genome for many cancers, several of them are also functionally linked to known oncogenes [5]. The MET gene is located on chromosome $7 \mathrm{q} 31$ in close proximity of another gene that has been implicated in cancer; the FAM $3 C$ locus is within $4.6 \mathrm{MB}$ of the $M E T$ gene on chromosome 7q31. FAM3C encodes for the interleukinlike EMT inducer (ILEI) protein, a secreted factor that can regulate tumor progression [6]. FAM $3 C$ was identified as an epithelial-to-mesenchymal transition (EMT)specific gene [7]. EMT occurs when epithelial cells lose their apical-basal polarity and cell-cell adhesion and switch to a migratory and invasive mesenchymal phenotype [8]. This process is vital during development and can be reactivated when required, such as during wound healing [9]. In cancer, EMT activities might be switched on transiently and reversibly to convert adherent epithelial tumor cells into motile and invasive mesenchymal cells [10]. In murine and human cellular models of breast, hepatocellular carcinoma, and lung cancer ILEI is required and sufficient to induce EMT and invasion in vitro and metastasis in vivo $[6,11,12]$. ILEI is overexpressed in several human tumors and shows altered subcellular localization, which is related to changes in the secretion levels of the protein [13]. ILEI localization strongly correlates with metastasis formation and survival in human breast and hepatocellular carcinomas $[6,11,12]$. In colorectal cancer, upregulation of ILEI protein expression correlates with EMT and poor prognosis [14].

However, whether increased expression of ILEI in some cancers is associated with increased $\mathrm{CN}$ of the $F A M 3 C$ locus is unclear. The proximity of FAM3C to the $M E T$ hotspot suggests that the two genes may be often co-amplified. The aim of this study was to investigate whether increased FAM $3 C \mathrm{CN}$ was evident in various cancers and reveal whether there was a relationship with $M E T$ amplification. Our results suggested a strong correlation between the copy number of the two genes. Further in vitro mechanistic investigations and xenograft experiments with combined counteraction of ILEI and c-MET activities suggest that c-MET and ILEI cooperate to increase the invasiveness of cancer cells.

\section{Methods}

\section{Materials and methods}

\section{Cell lines}

NCI-H1993 (CRL-5909), NCI-H441 (HTB-174), SKBR3 (HTB-30), MKN-45 (ACC409), OE33 (ACC706), MCF7 (HTB-22), MDA-MB-231 (HTB-26) and NIH3T3 (CRL1658) cell lines were obtained from ATCC (http://www. lgcstandards-atcc.org/en.aspx) or DSMZ (http://www. dsmz.de/), tested for mycoplasma infection on a regular basis using a commercial biochemical test (Lonza) and authenticated using STR profiling. All cells were cultured in Dulbecco's Modified Eagle's Medium/Nutrient Mixture F-12 Ham, 1:1 mixture supplemented with 10\% fetal calf serum (FCS), or in case of MKN45 with 20\% 
FCS. Upon serum withdrawal, FCS was replaced by $0.1 \%$ bovine serum albumin.

\begin{abstract}
Animals
Eight-twelve week-old female severe combined immunodeficiency disease (SCID) mice (Envigo, Italy) were used for xenograft experiments in this study. All animal work was done by following earlier protocols ethically approved by the Institutional Animal Care and Use Committee of the Medical University of Vienna and by the Austrian Bundesministerium für Bildung, Wissenschaft und Forschung (BMWFW-66.009/0081-WF/V/3b/2015).
\end{abstract}

\section{Human tumor samples}

Formalin fixed paraffin embedded primary tumor samples of advanced stage colorectal carcinoma were obtained from stored samples from de-identified patients treated in Kecskemet General Hospital, Hungary, who had previously provided informed consent for their use in clinical research.

\section{Genomic DNA isolation and qPCR-based determination of gene CNs from formalin fixed paraffin embedded human tumor samples}

Genomic DNA was isolated from non-stromal regions of 3-4 $10 \mu \mathrm{m}$ thick sections of formalin fixed paraffinembedded tumors of 49 advanced-stage colorectal carcinoma patients using the Gentra Puregene Tissue Kit (Qiagen) according the manufacturer's instructions. 60 ng of isolated genomic DNA was used as template in the quantitative real-time PCR reaction. All samples were done in triplicates and the MET and FAM3C copy numbers were derived by standardizing the input DNA to the control signal (TOP3A, chromosome 17p11) as described earlier [15]. The sequences of the primer pairs and probes for TOP3A and MET were as described in [16] using FAM as flourogenic label. For FAM3C the primers Hsp.FAM3C_F 5'-GTCACACTCTTGTGCCAG TCT-3' and Hsp.FAM3C_R 5'-GAGCAAAGGTCAGG GTTGAAAG-3' were used with the HEX-labeled probe Hsp.FAM3C_probe 5'-TCTGCAGCTTCAAATCCC CTCCTG-3' allowing duplex PCR with the TOP3A control gene.

\section{CN analysis of data in the TCGA database and human cancer cell lines}

Gene CNs of FAM3C, MET, EGFR, and FGFR1 were calculated using the genome sequencing datasets of several cancer entities in the TCGA database (https://www. cancer.gov/about-nci/organization/ccg/research/ structural-genomics/tcga). GISTIC 2.0 values were obtained from the Firehose system using Caleydo v3 software (caleydo.org): -2 , deep deletion; -1 , shallow deletion; 0 , diploid; 1 , gain; 2, amplification. RNA-seq data were obtained from Cancer Browser database (https://genome-cancer.ucsc.edu/).

Copy number $(\mathrm{CN})$ data of 200 tumor cell lines were generated using the GeneChip Human Mapping $250 \mathrm{~K}$ Nsp Arrays (Affymetrix) and subsequently analyzed on the Affymetrix ${ }^{\circ}$ Genotyping Console ${ }^{\mathrm{rm}}$ software (GTC) using the unpaired CNAT 4.0 analysis algotrythms. Gene copy number \pm 3 was considered as amplification, $<3$ as non-amplified.

Generation of stable cell lines expressing ILEI ShRNA vectors For ILEI and mammalian non-targeting control shRNA knock-down in NCI-H1993, NCI-H441, MKN45, OE33 and SKBR3 cells, MISSION shRNA lentiviral transduction particles (Sigma, St Louis, MS, USA) were used according to the manufacturer's instructions. Five shRNA sequences were pretested for ILEI knock-down (sh261 CCGGGATGCAAGTTTAGGAAATCTACTCGAGTAG ATTTCCTAAACTTGCATCTTTTTG, sh328 CCGG CCAGATATAAGTGTGGGATCTCTCGAGAGATCCC ACACTTATATCTGGTTTTTG, sh506 CCGGAGGAGA AGTATTAGACACTAACTCGAGTTAGTGTCTAATA CTTCTCCTTTTTTG, sh579 CCGGGCCATACAAG ATGGAACAATACTCGAGTATTGTTCCATCTTGTA TGGCTTTTTG and sh1767 CCGGCCTGTGTTTATC TAACTTCATCTCGAGATGAAGTTAGATAAACACA GGTTTTTG) and two were selected (sh261 and sh506) as the most efficient for later studies. In studies with only one shILEI cell line, "shILEI" indicates sh506. Stable cell lines were established using selection for puromycin resistance of transduced cells. ILEI expression was validated in whole cell lysates and conditioned medium (CM) by Western blotting.

\section{Western blot analysis}

Western blot analysis was performed as previously described [13]. Cells were treated with crizotinib (500 nM), savolitinib $(1 \mu \mathrm{M}$, both dissolved in DMSO) or DMSO for $24 \mathrm{~h}$, conditioned media $(\mathrm{CM})$ were collected after 24h. Anti-ILEI [6], anti-phospho cMET (\#3077), anticMET (\#3127), anti-phosphoErk1/2, anti-Erk1/2, anti-Ecadherin, anti-vinculin (Cell Signaling Technologies), anti- $\alpha$ tubulin, and anti- $\beta$ actin (Sigma) primary antibodies were used followed by enhanced chemiluminescent (ECL) detection using Chemidoc Touch (Bio-Rad) for digital capturing and ImageLab software (Bio-Rad) for visualization and quantification.

\section{${ }^{3} \mathrm{H}$-thymidine incorporation assay}

Cells were pretreated with different concentrations of crizotinib for $24 \mathrm{~h}$ and seeded in triplicates in 96 well plates in the presence of the same inhibitor concentrations. After $24 \mathrm{~h}$ of incubation, cells were labeled with $30 \mu \mathrm{Ci} / \mathrm{ml}$ methyl ${ }^{3} \mathrm{H}$-thymidine for $2 \mathrm{~h}$. Radioactive 
media was removed, cells were washed in phosphatebuffered saline (PBS) and trypsinized. Cells were fixed by Tomtec cell harvester (Tomtec Inc., USA) onto a waxembedded filtermat, and radioactive intensity was determined by a Wallac 1450 MicroBeta liquid scintillator (PerkinElmer Inc., USA). Results were normalized according to cell number.

\section{MTT assay}

Cells were plated in $100 \mu \mathrm{l}$ complete medium in 96-well flat bottom plates in the presence or absence of crizotinib (500 nM) or vehicle (DMSO) or increasing concentrations of PHA665752 (Sigma) and Savolitinib (Selleckchem). After $24 \mathrm{~h}$ (crizotinib) or $72 \mathrm{~h}$ (PHA665752, savolitinib) of incubation $10 \mu \mathrm{l}$ of $5 \mathrm{mg} / \mathrm{ml}$ 3-(4,5-dimethylthiazol-2-yl)-2,5-diphenyltetrazolium bromide (MTT) solution dissolved in PBS was added for $2 \mathrm{~h}$ followed by the addition of $100 \mu \mathrm{l}$ of solubilization solution (40\% dimethylformamide, $2 \%$ acetic acid, $16 \%$ sodium dodecyl sulfate, $\mathrm{pH} 4.7$ ) and rigorous shaking to dissolve the formazan crystals. The absorbance at 570 $\mathrm{nm}$ was determined using a Tecan plate reader (Tecan, Austria). Triplicate wells were assayed for each condition.

\section{Trans-well invasion assay}

Cells were pre-starved overnight in starvation or low (1\%) FCS medium and seeded in the same medium into transwell invasion chambers with $8 \mu \mathrm{m}$ pore size, coated with Matrigel (Corning Inc., USA) and pre-equilibrated with medium (50.000 cells/inlet, each condition in triplicates). The lower chamber of the trans-well unit contained conditioned medium of NIH3T3 cells or human HGF (40 ng/ $\mathrm{ml}$ ) as attractant. For c-MET inhibition, medium was supplemented with $500 \mathrm{nM}$ crizotinib both in the upper and lower chambers. Cells were allowed to invade for $24 \mathrm{~h}$, non-invaded cells were removed from the upper side of the inlets, and cells were fixed and stained with 4',6-diamidino-2-phenylindole (DAPI). Total numbers of invasive cells were counted using fluorescent microscopy imaging followed by ImageJ analysis.

\section{RNA isolation and real-time quantitative $P C R$ analysis}

Total RNA was extracted, reverse transcribed and cDNA was amplified with primers for the genes $M M P 9, M M P 2$ and $C D H 1$ as described earlier [17].

\section{Gelatin zymography}

For MMP-9 and MMP-2 detection in the CM of NCIH441 and NCI-H1993 shCont and shILEI cells upon HGF stimulus with or without crizotinib treatment cells were plated in 6-well plates. At 70-80\% confluency, cells were crizotinib- $(500 \mathrm{nM})$ or DMSO-treated for $30 \mathrm{~min}$ followed by medium change to FBS-free media with continued crizotinib supply and the addition of human HGF $(40 \mathrm{ng} / \mathrm{ml})$. 24-h CM were collected and concentrated as described for Western blot analysis and all samples were adjusted to the same protein concentration followed by equal volume gel loading.

For MMP-9 and MMP-2 detection in protein extracts, NCI-H441 and NCI-H1993 shCont and shILEI snapfrozen tumor pieces with or without crizotinib treatment were homogenized in lysis buffer (see Western blot analysis for composition), total protein concentration was determined and equal protein amounts were loaded.

A $7.5 \%$ acrylamide gel was prepared with $2 \mathrm{mg} / \mathrm{ml}$ final concentration of gelatin; samples were loaded in nonreducing sample buffer without boiling. After running, the gel was washed twice for $30 \mathrm{~min}$ each in washing buffer (2.5\% Triton X-100, $50 \mathrm{mM}$ TRIS-HCl, pH 7.5, 5 $\mathrm{mM} \mathrm{CaCl} 2,1 \mu \mathrm{M} \mathrm{ZnCl}_{2}$ ), rinsed for $10 \mathrm{~min}$ in incubation buffer (1\% Triton X-100, $50 \mathrm{mM}$ TRIS-HCl, pH 7.5, 5 $\mathrm{mM} \mathrm{CaCl}, 1 \mu \mathrm{M} \mathrm{ZnCl}_{2}$ ) at $37^{\circ} \mathrm{C}$ followed by incubation in fresh incubation buffer for $24-40 \mathrm{~h}$ at $37^{\circ} \mathrm{C}$. The gel was stained in staining solution $(0.5 \%(\mathrm{w} / \mathrm{v})$ Coomassie Blue, $10 \%$ acetic acid, $40 \%$ methanol) for $1 \mathrm{~h}$, rinsed with water and incubated in destaining solution $(10 \%$ acetic acid, $40 \%$ methanol) until white bands were clearly visible. $10 \mathrm{ng}$ of recombinant MMP-9 (Thermofisher Scientific) was loaded as positive control. Gels were quantified using ImageJ.

\section{Immunohistochemistry}

Immunohistochemistry on paraformaldehyde fixed, paraffin embedded, $3 \mu \mathrm{m}$ thick sections of mouse tumor tissues was performed manually using anti-Ki67 (1:2000), anti-CD31 (1:200) (abcam), anti-cleaved Caspase3 (1: 1000), anti-phospho cMET (1:1000), anti-cMET (1: 1000), anti-MMP9 (1:1000) (Cell Signaling Technologies), anti-E-cadherin (1:1000) (BD Biosciences) and anti-ILEI (1:1000) [6] primary antibodies and Lab Vi$\operatorname{sion}^{\text {Tw }}$ UltraVision $^{\text {Tx }}$ LP Detection System (Thermo Scientific) with 3,3'-diaminobenzidine (DAB) substrate (Dako) for detection according to the manufacturer's instruction. Cell nuclei were visualized by hematoxylin staining. Histological samples were scanned using a Pannoramic MIDI slide scanner (3D Histech) with a 40X objective. Subsequently, quantification of immunomhistochemistry was performed by the histomorphometric software package Tissue Studio ${ }^{\circ}$ (Definiens AG). The Ecadherin membrane score was obtained by the formula: $3 \mathrm{x}$ ratio of high membrane staining intensity $+2 \mathrm{x}$ ratio of medium membrane staining intensity $+1 \mathrm{x}$ ratio of low membrane staining intensity, giving a range of 1 to 3 .

\section{Experimental mouse xenografts}

Mouse xenografts were established with subcutaneous injection of $1.7 \times 10^{6}$ control (shCont) or ILEI KD 
(sh506) NCI-H1993 and NCI-H441 cells into 8-12-week old female SCID mice $(n=4)$. Injected mice were distributed into randomized cohorts and vehicle and compound treatment started as the mean tumor size reached $100 \mathrm{~mm}^{3}$. Crizotinib (LC Laboratories) was applied orally $(50 \mathrm{mg} / \mathrm{kg}$, dissolved in 5\% DMSO, 10\% ethanol and 10\% Cremophor) in a 5-days treatment 2-days pause protocol. For NCI-H1993 tumors, at the time point of sacrifice of vehicle-treated mice, crizotinib treated animals were monitored for an additional 11 days without further supply of the compound. Tumors were measured regularly by a caliper and tumor volume was calculated by the formula $a \times b^{2} / 2$ ( $a$ for the major and $b$ for the minor tumor diameter). The tumors were dissected, and tumor mass was determined 40-50 days after injection.

\section{Statistical analysis}

Data are expressed as the mean \pm standard error of the mean (SEM) where applicable. Data normality was checked using Shapiro-Wilk and Kolmogorov-Smirnov tests. Statistical significance was determined by unpaired two-sided Student's $t$-test, one-way and two-way analysis of variance (ANOVA) tests and Kruskal-Wallis tests followed by Dunn's multiple testing adjustment using Graph Prism software (version 5.0). Survival differences were calculated using log rank tests. $p<0.05$ was considered significant. Kendall's tau-b tests and Chi-square tests were calculated using $\mathrm{R}$ software (version 3.2.1). Alluvial plots were generated using Caleydo 3.0 software [18].

\section{Results}

Gene amplification of $F A M 3 C$ and $M E T$ is tightly linked in several human carcinomas and correlates with increased gene expression and poor prognosis

To determine the frequency of $\mathrm{CN}$ amplification of the $F A M 3 C$ and $M E T$ genes, we investigated a variety of datasets of different cancer entities from the TCGA database. Connections in gene function and physical location of the two genes were uncoupled by analysis of two additional RTKs with dedicated driver functions in the progression of many malignancies: Epidermal growth factor receptor (EGFR), located on the $\mathrm{p}$ arm of the same chromosome and fibroblast growth factor receptor 1 (FGFR1) located on chromosome 8. Of 501 lung squamous cell carcinoma (LUSC) cases, 8 (1.9\%) indicated $M E T$, out of these $6(1.4 \%)$ also FAM3C CN amplifications (Fig. 1a). Of 516 lung adenocarcinoma (LUAD) cases, 18 (3.5\%) had MET and 9 (1.7\%) FAM3C CN amplification, 8 deriving from the MET amplification group (Fig. 1d). 1480 hepatocellular carcinoma (LIHC) cases had in $11(0.7 \%)$ and $6(0.4 \%)$ cases with amplification for the $M E T$ and $F A M 3 C$ genes, respectively, in 5 cases with a shared amplification for both, 615 colorectal adenocarcinoma (COADREAD) cases showed $\mathrm{CN}$ amplification in 1 case $(0.2 \%)$ shared for both $M E T$ and FAM $3 C$ and 1080 breast cancer (BRCA) cases had CN amplification in 10 cases $(0.9 \%)$ for both $M E T$ and $F A M 3 C, 7$ sharing amplification for both (Supplemental Fig.S1A). Correlation analyses showed that the FAM3C gene CNs were tightly correlated with CNs of $M E T$ but not with the distant EGFR or unlinked FGFR1 genes in all analyzed data cohorts for LUSC (Fig. 1a), LUAD (Fig. 1d), LIHC, COADREAD, and BRCA (Supplemental Fig.S1A), indicating that co-amplification might be a consequence of chromosomal proximity.

To test if genomic amplification influenced gene expression, $M E T$ and $F A M 3 C$ gene $\mathrm{CNs}$ were then compared to mRNA levels and relapse-free survival of LUSC and LUAD patients from the TCGA database. The analysis showed that $\mathrm{CNs}$ of both genes significantly correlated with gene expression levels, patients with strong $M E T$ and/or FAM3C amplification showing the highest expression of these genes (Fig. $1 \mathrm{~b}$ and e). Patients with $M E T$ and/or FAM3C amplification also had a significantly worse survival compared to the pooled cohort of patients with deletions, normal, or slight gain in the $\mathrm{CN}$ of the two gene loci, albeit low case number and early loss on patient follow-up prevented a proper analysis on the survival of LUSC FAM 3 C amplified patients (Fig. 1c and f). These data indicate that the FAM $3 C$ and $M E T$ genes are frequently co-amplified in human cancers contributing to increased gene expression and poor survival.

We then tested the linkage of the two genes on genomic DNA isolated from formalin fixed paraffin-embedded tumors of 49 advanced-stage colorectal carcinoma patients by qPCR. Over $72 \%(24 / 33)$ of the FAM3C-and/or $M E T$-amplified tumors showed a co-amplification of the two genes, further supporting the tight linkage of these loci (Supplemental Fig.S1B). In addition, cluster and correlation analysis of $F A M 3 C$ amplification with available clinicopathological parameters elucidated a significant enrichment of FAM3C amplification in patients with extramural venous invasion (EMVI) (Supplemental Fig.S1B, C). EMVI, the spreading of cancer cells into the nearby blood vessels, is an invasive characteristic connected to worse prognosis. Although ILEI has not been linked so far to EMVI, our finding is in accordance with the described function of ILEI in inducing EMT and invasion and reflects that FAM $3 C$ amplification might affect gene function resulting in a clinically worse outcome. So, these results support the database analysis showing frequent co-amplification of $M E T$ and FAM3C and the likelihood of poor survival rates in patients with increased CNs of these genes.

Increased CNs of FAM3C and MET are tightly linked and frequently present in multiple human cancer cell lines To evaluate if our findings on FAM $3 C-M E T$ coamplification in human primary tumors can be 


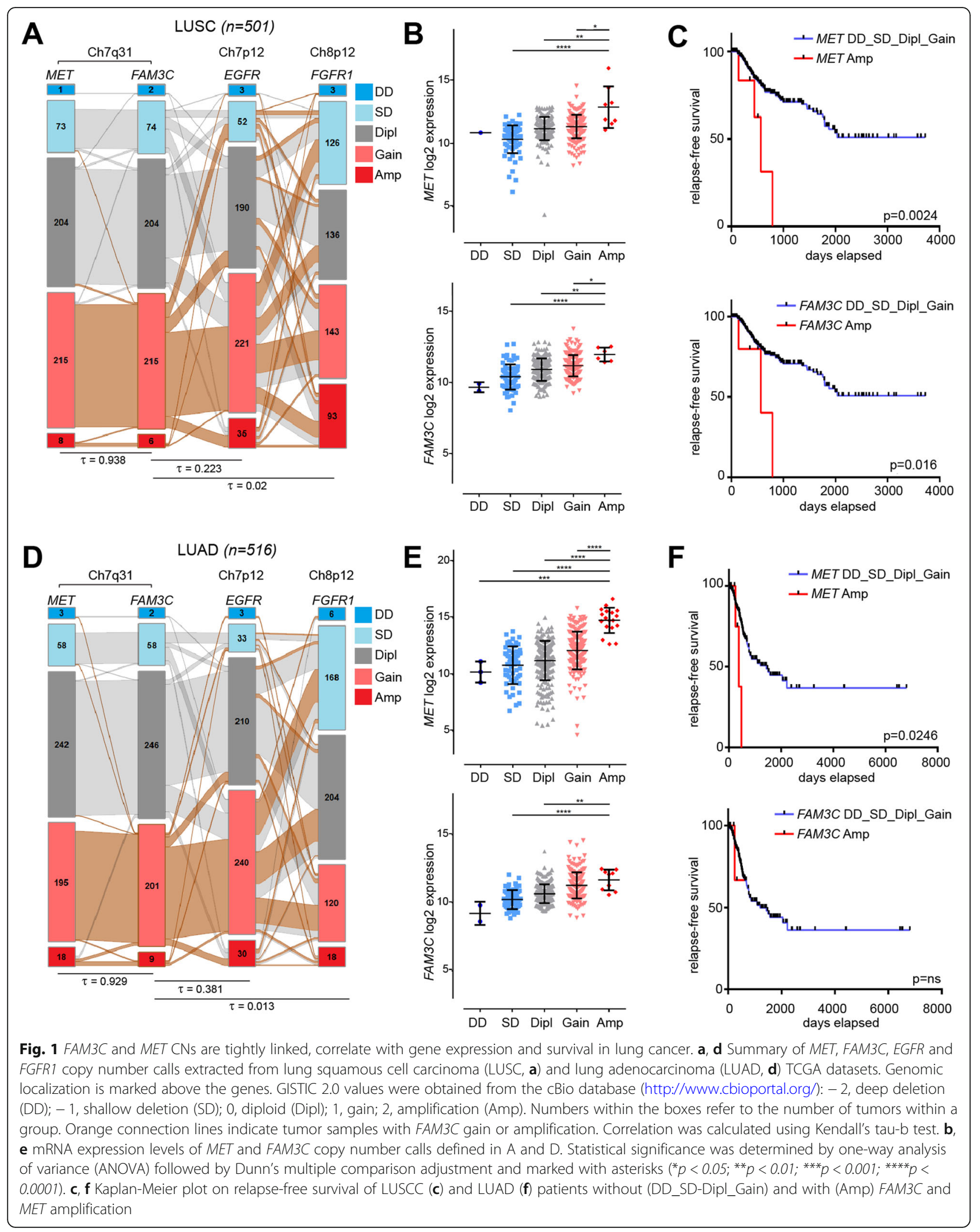


recapitulated in cultured human cancer cell lines, FAM $3 C$ and MET gene CNs were determined in a panel of 200 human cancer cell lines of diverse tissue origins by microarrays and analysed using the CNAT 4.0 analysis algotrythms in the GTC analysis (Fig. 2a). Increased $\mathrm{CN}$, using a cut-off of $\mathrm{CN} 3$ or higher, of both genes was present in cell lines of all tumor entities at a frequency of $47 \%$ on average. The frequency varied in the different cancer types, breast and lung cancer had the lowest (25 and 30\%) and melanomas the highest (76\%) (Fig. 2a, left panel). Importantly, over $90 \%$ of the cell lines with an increased $\mathrm{CN}$ for at least one of the genes showed an increase for both loci (Fig. 2a, right panel), confirming that the amplification event of the two genes is tightly coupled and indicating that cancer cell lines representatively illustrate in vitro the FAM $3 C-M E T$ coamplification characteristics of primary tumors.

Of the 85 cell lines with increased $\mathrm{CN}$ for both $F A M 3 C$ and $M E T$, we selected five for detailed investigation (Fig. 2b). These were a pair of gastrointestinal cancer cell lines (MKN45 and OE33), a pair of lung adenocarcinoma cancer cell lines (NCI-H1993 and NCIH441), and a breast cancer cell line (SKBR3). One cell line in each of the gastrointestinal and lung adenocarcinoma pairs had previously been described as sensitive and the other as resistant to the c-MET inhibitor PHA665752 [19]. Both these pairs of cell lines expressed high levels of ILEI and c-MET (Fig. 2c) as compared to control samples from non-metastatic MCF7 and the metastatic MDA-MB-231 human breast cancer cell line lacking FAM $3 C$ and $M E T$ amplifications but with upregulated ILEI expression as a characteristics of metastatic capacity [4]. These cell lines with high expression levels also secreted ILEI into the CM during culture. In SKBR3 cells, c-MET expression is absent despite locus amplification due to epigenetic silencing [5]. Accordingly, this cell line did not express c-MET and interestingly, though not silenced, ILEI expression was also only moderate and secretion almost absent despite increased gene $\mathrm{CN}$ (Fig. 2c).

Stable ILEI knock-down does not influence proliferation capacity and sensitivity towards c-MET-inhibitor induced proliferation arrest

Since there are no specific pharmacological inhibitors for ILEI available, we mimicked ILEI inhibition by RNA interference-mediated (RNAi) stable knock-down (KD) of the protein expression. Both intracellular and secreted

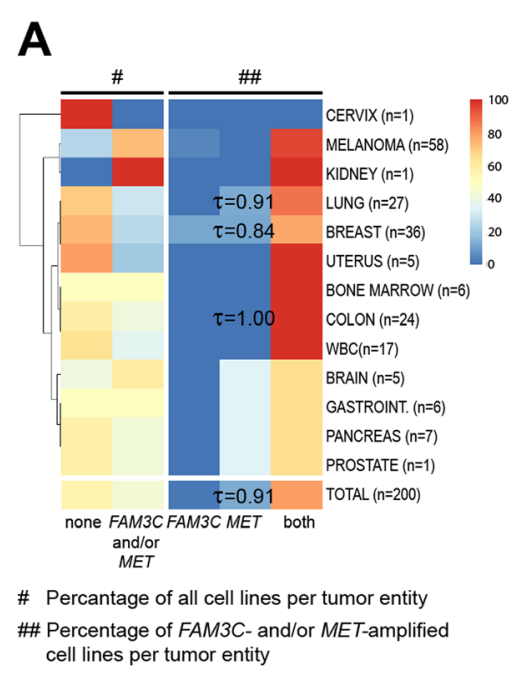

B

\begin{tabular}{|c|c|c|c|c|}
\hline \multirow{2}{*}{ cell line } & \multicolumn{2}{|c|}{ copy number (CN) } & \multirow{2}{*}{$\begin{array}{c}\% \text { viability } \\
\text { after } \\
\text { PHA665752b }\end{array}$} & \multirow{2}{*}{ tissue origin } \\
\hline & $\mathrm{CMET}^{\mathrm{a}}$ & ILEI & & \\
\hline $\mathrm{NCl}-\mathrm{H} 1993$ & $14(8.7)$ & 14 & 42 & lung (adenocarcinoma) \\
\hline $\mathrm{NCl}-\mathrm{H} 441$ & $>4(3)$ & $>4$ & 93 & lung (adenocarcinoma) \\
\hline MKN45 & $12(6.5)$ & 12 & 25 & gastric (stomach) \\
\hline OE33 & $>4(6.1)$ & $>4$ & 53 & oesophageal \\
\hline SKBR3 & $>4(4.2)$ & $>4$ & 1.05 & breast \\
\hline
\end{tabular}

C

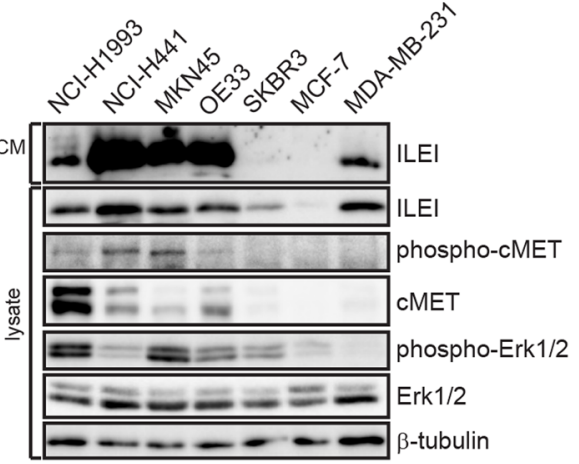

Fig. 2 Increased FAMBC and MET CNs are frequently combined and present in human cancer cell lines. a Percentage of cell lines bearing increased FAM3C and/or MET gene CNs (amplification cut-off was set CN $\geq 3$ ) determined by GeneChip Mapping Assay in a panel of 200 cancer cell lines of diverse tissue origin. Left panel (\#), percentage of cell lines with increased FAM3C and/or MET CNs; right panel (\#\#), percentage of combined FAM3C and MET CN increase within the FAM3C-and/or MET-amplified cell lines. Number of analyzed cell lines for each tumor entity are listed on the right side. Correlation, marked for LUNG, BREAST, COLON and TOTAL, was calculated using Kendall's tau-b test. WBC; white blood cell. b Short-list of five selected human tumor cell lines from panel A with increased FAM3C and MET CNs and different sensitivity to the MET inhibitor PHA665752. MET CNs in brackets and \% viability after PHA665752 treatment are taken from [19]. c Western blot analysis of ILEI secretion into conditioned media (CM) and expression within the cells and MET and Erk expression and activity in above five selected cell lines. MCF-7 and MDA-MB-231 human breast cancer cell lines were used as normal FAM3C CN controls with low and high ILEl expression 
ILEI protein levels showed an apparent reduction by two independent shRNAs in all five cell lines, which was most evident in the levels of the functionally relevant secreted form in the CM (Fig. 3a). For cMET blockade, we used crizotinib, a small-molecule tyrosine kinase inhibitor that efficiently inhibits c-MET, anaplastic lymphoma kinase 5 (ALK5) and ROS1 and is approved by the FDA for treatment of ALK-rearranged NSCLC [4]. As none of the selected cell lines including the two lung adenocarcinoma lines NCI-H1993 and NCI-H441 harbored an ALK rearrangement, inhibitor effects were expected to occur primarily due to c-MET inhibition. Since SKBR3 cells did not express c-MET despite of a $M E T$ and FAM3C amplification, they served as a control to monitor the potential influence of ALK5 and ROS1 targeting effects of crizotinib.

First, we analyzed the dose-dependency of crizotinib on the proliferation capacity of the five selected cancer cell lines. The OE33 cell line that had previously shown resistance to another small molecule c-MET inhibitor displayed sensitivity to increasing concentrations of crizotinib in proliferation capacity (Fig. $3 \mathrm{~b}$ ), comparable to the sensitive cell lines NCI-H1993 and MKN45. NCIH441 and SKBR3 tolerated high doses of the drug without remarkable drop in their proliferation rate or viability (Fig. 3b), confirming their described resistance towards MET inhibitors [19]. ILEI KD did not influence the proliferation behavior of the selected five cell lines (Fig. 3c). Furthermore, ILEI KD also did not influence the sensitivity of these cells towards crizotinib-induced growth arrest (Fig. 3c), indicating that ILEI does not affect proliferation and does not influence c-METdependent regulation of proliferation. To address any concerns of the polypharmacological action of crizotinib, which inhibits other targets such as ALK5, we also investigated the action of two additional c-MET inhibitors: PHA665752 and savolitinib. The results showed similar effects on cell viability as crizotinib (Supplemental Fig.S2).

\section{ILEI KD impairs both c-MET-independent and c-MET-} dependent invasion of cancer cells with FAM 3 C and MET $\mathrm{CN}$ gains

Next, we investigated invasiveness and its sensitivity to c-MET and ILEI signaling inhibition in the five selected cancer cell lines. First, we tested c-MET-independent invasive capacity in an in vitro trans-well invasion assay by using NIH3T3 CM as chemoattractant, as murine HGF produced by these cells does not cross-activate the human c-MET receptor [20, 21]. ILEI KD strongly impaired the invasiveness of all five cancer cell lines, whereas invasion capacity was not influenced by crizotinib treatment (Fig. 4a). This supported the view that ILEI signaling induces invasiveness. To test the influence of ILEI on c-MET induced invasion, the same assay was performed this time using human HGF as chemoattractant (Fig. 4b). As expected, crizotinib efficiently inhibited HGF-induced invasion in the four cMET-expressing cell lines. Importantly, ILEI KD also significantly impaired HGF-induced invasiveness in all cMET-expressing cells, indicating that ILEI might be a contributing factor in c-MET-driven cellular invasion. ILEI KD derivatives of NCI-H441 and OE33, even showed an increased sensitivity towards crizotinib with invasion almost completely eliminated, suggesting that ILEI depletion might have an additive inhibitory effect to crizotinib in these cells.

In summary, stable ILEI KD efficiently reduced both c-MET-dependent and c-MET-independent invasion in all tested cells.

While ILEI does not influence c-MET signaling activity, cMET acts on ILEl signaling activity by regulating ILEI secretion

As c-MET and ILEI interact during c-MET-dependent invasion, we tested the possibility of the two signaling pathways being linked by investigating the effect of crizotinib and ILEI KD on c-MET signaling. Crizotinib efficiently inhibited c-MET autophosphorylation in all four c-MET-expressing cell lines (Fig. 5a). Activation of Erk, an important downstream effector of c-MET, was also significantly reduced upon drug treatment in these cells, whereas it remained unaltered in SKBR3 cells, which do not express c-MET (Fig. 5a). Knock-down of ILEI did not have an influence on the expression and activation levels of c-MET and Erk (Fig. 5a). Similarly, c-MET inhibition did not influence ILEI expression levels in the tested cell lines (Fig. 5a), indicating that c-MET and ILEI expression is not cross-regulated. Importantly, however, crizotinib decreased the secretion of ILEI in all c-Met expressing cell lines, but not in SKBR3 cells (Fig. 5a). Similar results were also found with the c-MET specific inhibitor savolitinib (Supplemental Fig.S3A). This suggests that c-MET may positively regulate ILEI secretion.

\section{Elevated expression and secretion of MMPs upon c- MET activation depends on ILEI and the two pathways cooperate in E-cadherin repression}

To uncover potential mechanisms by which c-MET and ILEI might cooperate to increase invasiveness, we next investigated markers of invasion. MMPs remodel the extracellular matrix (ECM) and are activated during invasion to ease the movement of cancer cells [22]. So, we explored the mRNA expression levels of two prominent MMPs, MMP-2 and 9 in response to HGF in the five cell lines. Of note, none of the five cells showed expression of both of these MMPs; NCI-H441, MKN45 and OE33 expressed only MMP-9, whereas NCI-H1993 and 


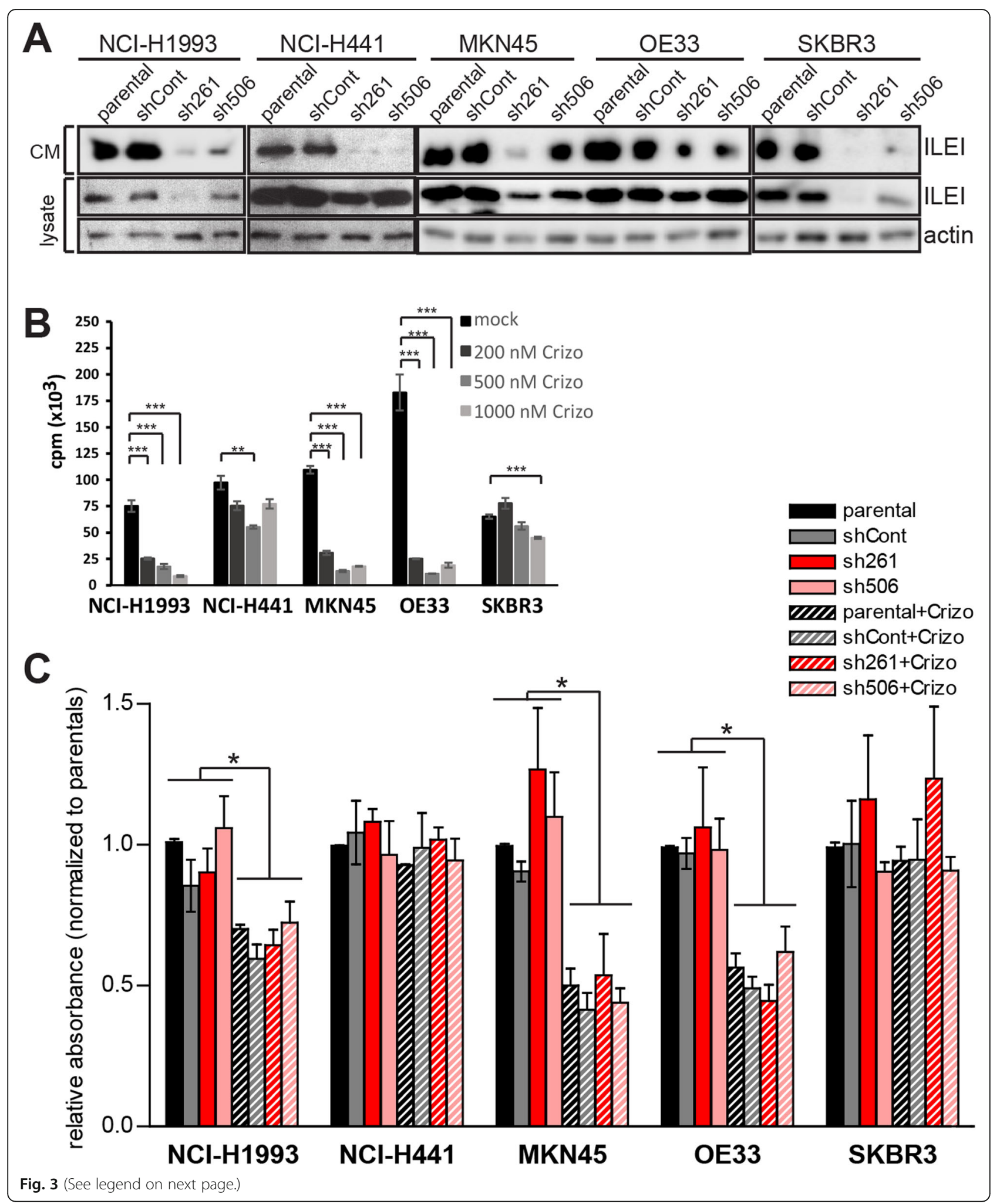


(See figure on previous page.)

Fig. 3 ILEI KD does not influence proliferation and sensitivity towards C-MET-inhibiton-induced arrest in FAM3C-MET-amplified cancer cells. a Western blot analysis of ILEl secretion into conditioned media (CM) and expression in the five selected cell lines (parental) and their non-targeting (shCont) and ILEI (sh261 and sh506) shRNA KD derivatives. The two independent ILEI KD lines sh261 and sh506 are named based on the starting position of the shRNA targeting site in the ILEI mRNA. $\mathbf{b}$ Proliferation behavior of the five tumor cell lines in the presence of increasing concentrations of crizotinib determined by thymidine incorporation assay. Error bars represent SEM of three independent replicates. $\mathbf{c}$ in vitro proliferation capacity of the five selected human tumor cell lines and their control and ILEI shRNA KD derivatives after $24 \mathrm{~h}$ vehicle or crizotinib (500 nM) treatment determined by MTT assay. Each cell line is normalized to its own parental vehicle-treated control. Error bars represent SEM of three to six independent assays. Statistical significance was determined by one-way ANOVA and marked with asterisks $\left({ }^{*} p<0.05 ;{ }^{* *} p<0.01\right.$; $\left.{ }^{* * *} p<0.001\right)$

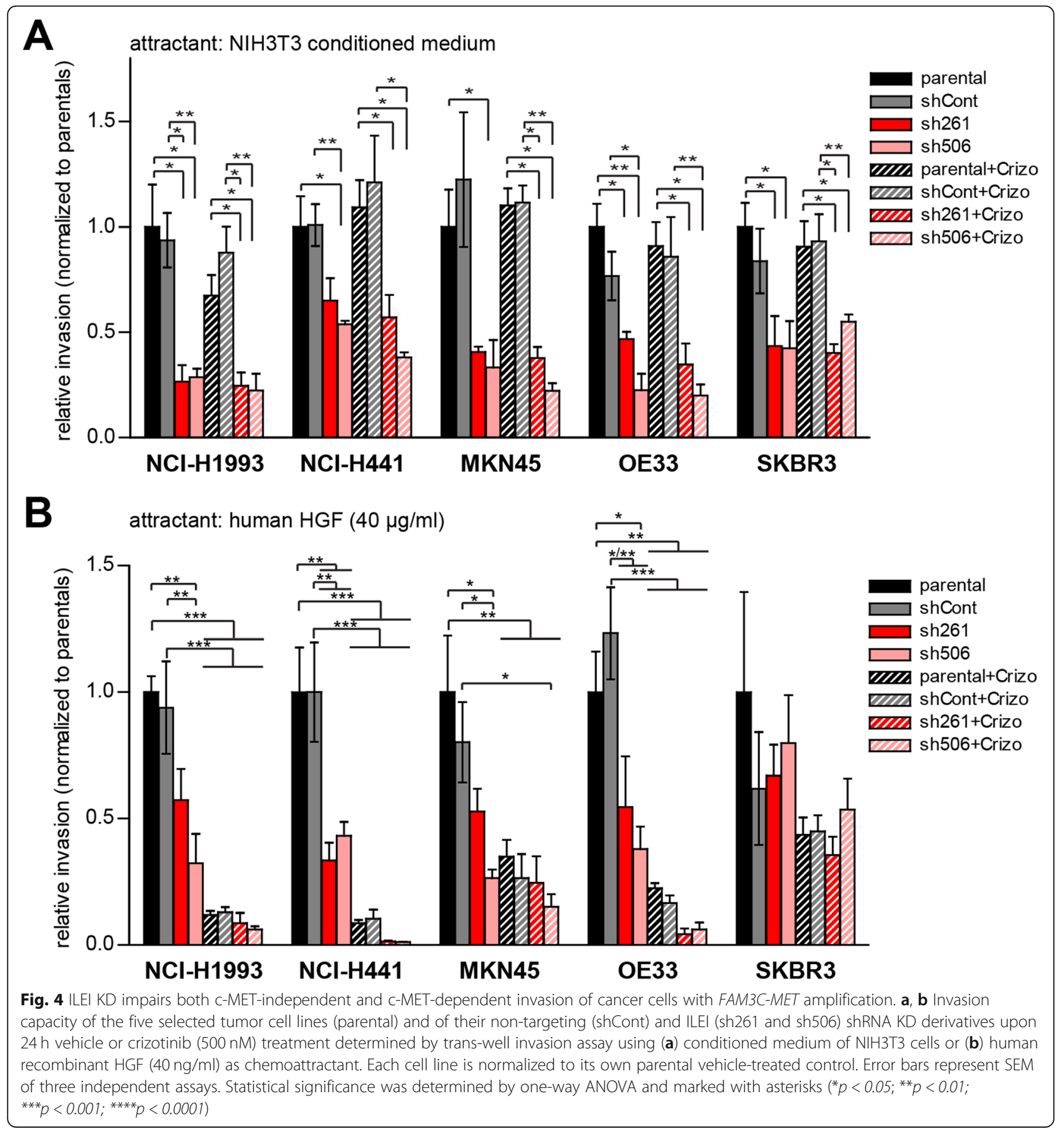




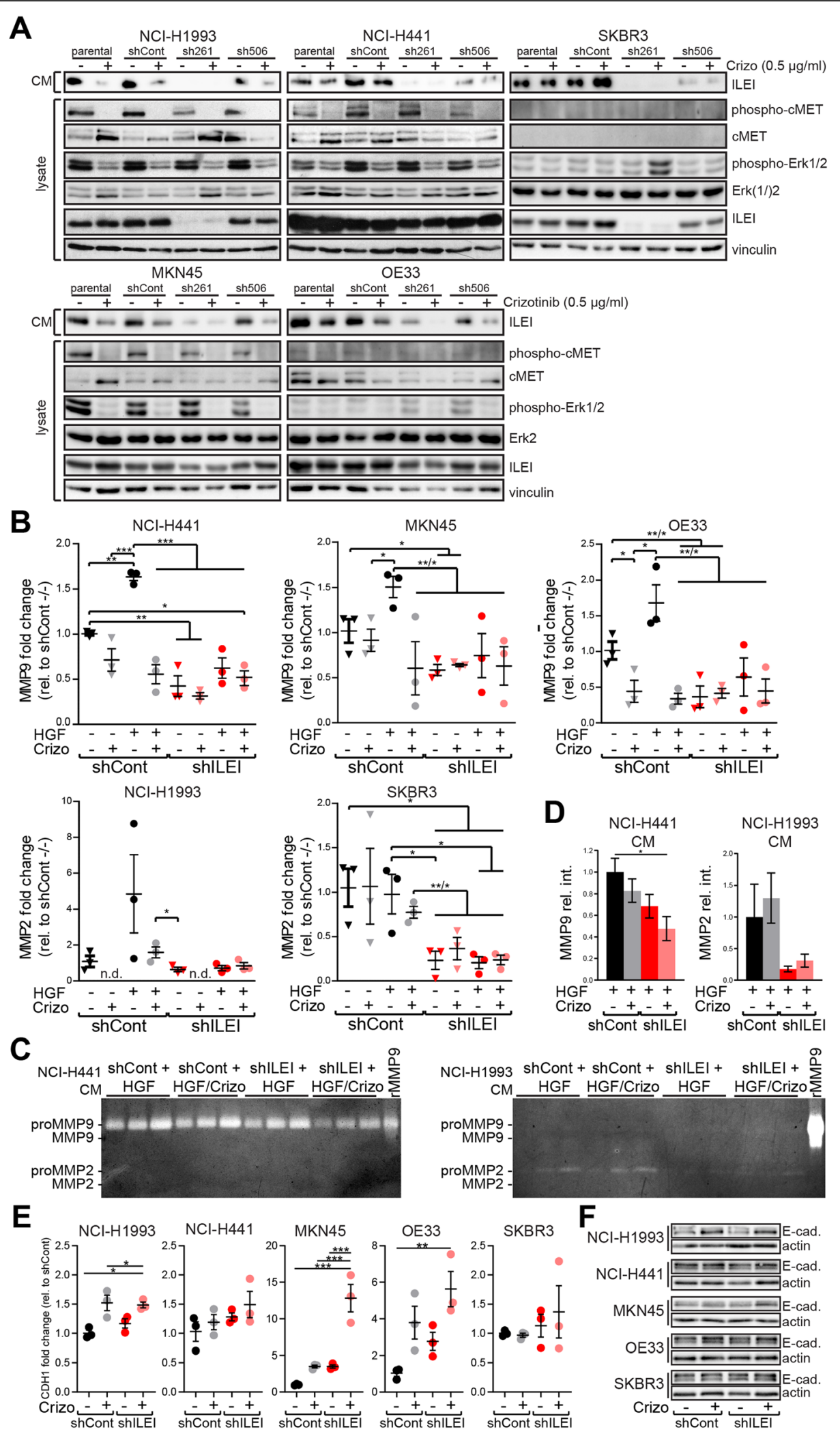

Fig. 5 (See legend on next page.) 
(See figure on previous page.)

Fig. 5 HGF-induced expression and secretion of MMPs requires ILEI, efficient ILEI secretion requires c-MET signaling. a Western blot analysis of ILE secretion and expression, and c-MET and Erk activity and expression in the five selected cell lines (parental) and their control (shCont) and ILEI KD (sh261 and sh506) derivatives after crizotinib (500 nM) treatment for $24 \mathrm{~h} . \mathbf{b}$ qPCR analysis of MMP-9 (for NCl-H441, MKN45 and OE33) and MMP-2 (for NCl-H1993 and SKBR3) mRNA expression in control (shCont) and ILEI KD (shlLEl) cells after $24 \mathrm{~h}$ of HGF treatment (40 ng/ml) in the absence or presence of crizotinib $(500 \mathrm{nM})$. Data are normalized as fold change to untreated control cells. Error bars represent SEM of three independent experiments. Statistical significance was determined by one-way ANOVA. c Secretion of MMP-9 and MMP-2 by control (shCont) and ILEl KD (shlLEl) NCl-H441 and NCl-H1993 cells treated with HGF $(40 \mathrm{ng} / \mathrm{ml})$ for $24 \mathrm{~h}$ in the absence or presence of crizotinib (500 nM) determined by gelatin zymography from harvested conditioned medium. The three lanes of each treatment group represent samples of three independent assays. Recombinant pro-MMP-9 was used as assay control. $\mathbf{d}$ Quantification of the gelatin zymography gels shown in C. Relative differences in secreted MMP-9 and MMP-2 levels were determined by ImageJ analysis and normalized to HGF treatment-induced control cells. Error bars represent SEM of three independent experiments. Statistical significance was determined by Student's t-test. e qPCR analysis of E-cadherin mRNA expression (CDH1) in NCl-H1993, NCl-H441, MKN45, OE33 and SKBR3 control (shCont) and ILEI KD (shILEl) cells treated or non-treated with crizotinib $(500 \mathrm{nM})$ for $24 \mathrm{~h}$. Data are normalized as fold change to untreated control cells. Error bars represent SEM of three independent experiments. Statistical significance was determined by one-way ANOVA and marked with asterisks $\left({ }^{*} p<0.05 ;{ }^{* *} p<0.01\right)$. f Representative Western blot analysis of E-cadherin expression in the control (shCont) and ILEI KD (shILEl) derivatives of the five selected cell lines after crizotinib (500 nM) treatment for $24 \mathrm{~h}$

SKBR3 cells only MMP-2. Firstly, ILEI KD lead to decreased expression of MMP-9 or MMP-2 in all five cell lines, confirming its role in invasion and MMP expression (Fig. 5b) and [17]. Secondly, in NCI-H441, MKN45, and OE33 cells HGF induced expression of MMP-9 mRNA and this expression was inhibited by crizotinib supporting the view that c-MET-dependent invasion activates MMP-9 (Fig. 5b) and [23, 24]. Even more importantly, however, in ILEI KD derivatives of these cells HGF treatment was not able to elevate MMP-9 mRNA expression (Fig. 5b). This result suggests that c-METmediated increased expression of MMP-9 mRNA during invasion is dependent on ILEI. A similar pattern was seen in NCI-H1993 cells for MMP-2 mRNA expression, but not in SKBR3 cells, where c-MET is not expressed and hence, HGF did not increase MMP-2 mRNA levels (Fig. 5b). As MMPs act on ECM, their activity is dependent upon secretion, so we analyzed the effect of c-MET and ILEI signaling inhibition on MMP secretion. For easier detection of inhibitory effects, high baseline secretion was ensured by HGF trigger. The secretion of MMP-9 from NCI-H441 cells decreased slightly with crizotinib treatment or ILEI KD, and the combination of the two lead to a significant reduction (Figs. 5c, d). In NCI-H1993 cells ILEI KD was sufficient for remarkable reduction of MMP-2 secretion (Fig. 5c, d). The specificity of these results to c-MET inhibition was also tested with savolitinib with similar results (Supplemental Fig.S3b). Overall, these results suggest that both c-MET and ILEI contribute to efficient secretion of MMPs in a cooperative and partially complimentary manner, c-Met most probably acting indirectly, via regulating ILEI secretion.

Another important marker of invasion and EMT status is the loss or reduction of the cell adhesion molecule Ecadherin [25]. Therefore, we checked potential changes in the levels of E-cadherin mRNA (CDH1) and protein upon crizotinib treatment and ILEI KD in each of the cell lines used in this study, and found high variance according to the cell type (Fig. 5e, f). On the one hand, in the crizotinib-resistant NCI-H441 and SKBR3 cells no significant differences in CDH1 mRNA expression levels were observed (Fig. 5e). On the other hand, NCI-H1993, MKN45 and OE33 cell lines showed a significant c-MET and ILEI mediated regulation of CDH1 transcription. All three cell lines showed increase in CDH1 mRNA levels in ILEI KD derived cells or with crizotinib, and combination of these conditions resulted in more stable or superior effects. Cells tested with savolitinib showed similar results (Supplemental Fig.S3c). E-cadherin protein levels showed a similar trend of differences as of transcription (Fig. 5f). This once again suggests a cooperation between c-MET and ILEI in the regulation of E-cadherin transcription, however, these data also point out that cancer cells show very different sensitivity towards this regulation.

\section{Combined ILEI KD and crizotinib treatment significantly reduced the outgrowth of $\mathrm{NCl}-\mathrm{H} 441$ and $\mathrm{NCl}-\mathrm{H} 1993$ tumor xenografts}

To assess the in vivo relevance of the above findings, we next investigated the growth of tumor xenografts induced by NCI-H441 and NCI-H1993 cells and their ILEI KD derivatives in the presence and absence of crizotinib treatment in a mouse model. The original rationale was to compare the effect of ILEI KD on the growth capacity of crizotinib-sensitive vs crizotinib resistant tumors. Because we were expecting a very low response of the NCI-H441 cell line towards crizotinib we did not plan a withdrawal step for that cell line, while the NCI-H1993 cell induced xenografts were evaluated for crizotinib withdrawal for an additional 11 days. However, in accordance with some earlier findings [26], we observed that inhibition of c-MET with crizotinib reduced the growth and tumor mass of both of the xenografts (Fig. 6a, b, j, k), indicating that crizotinib was able to 


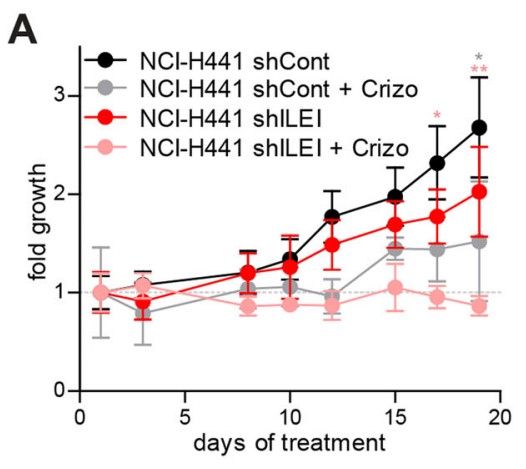

B
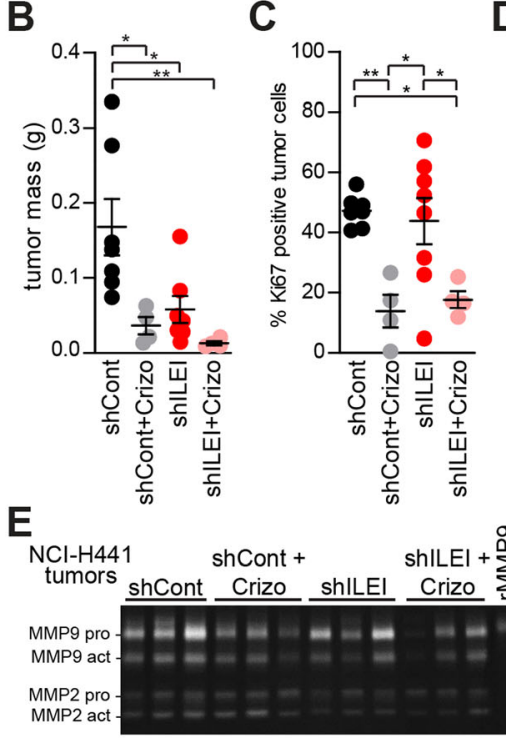

F
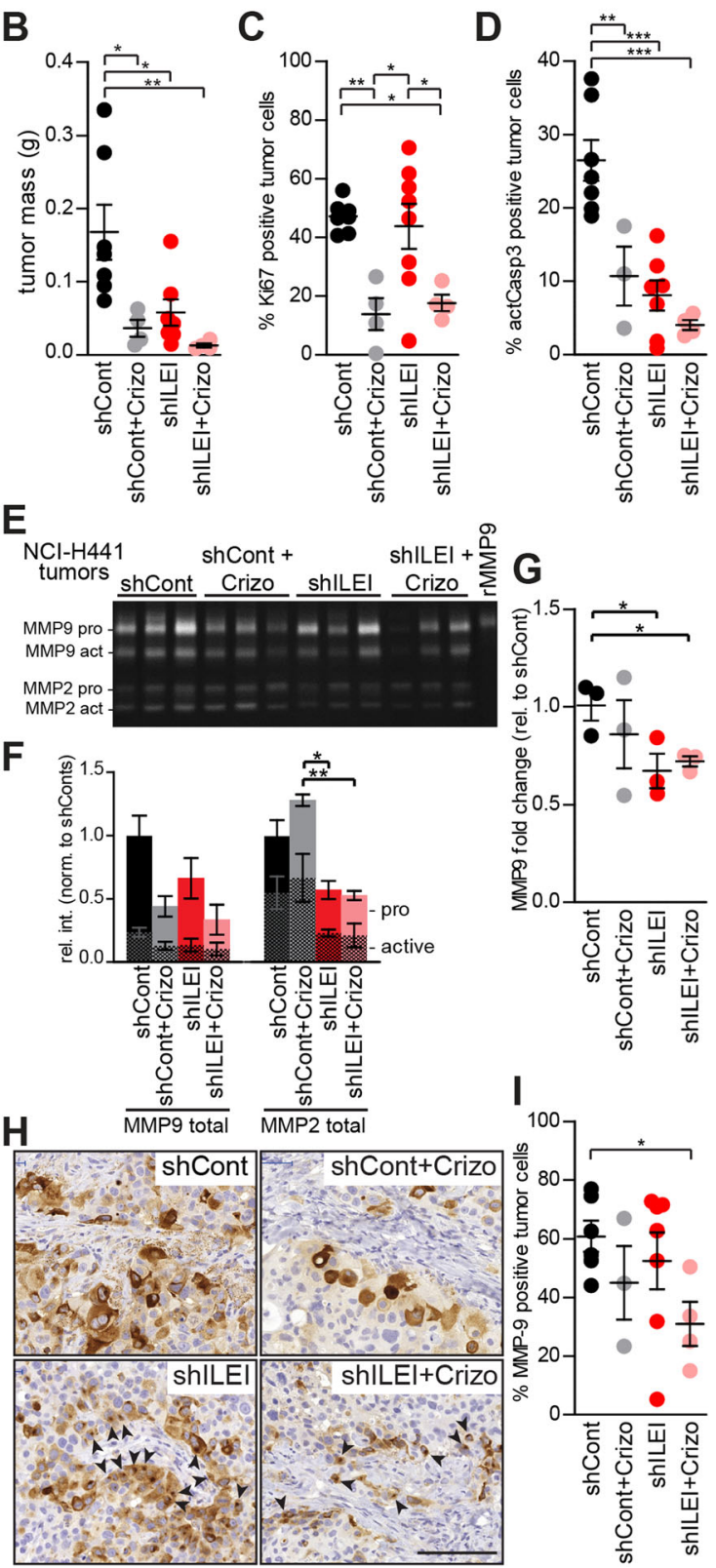

$\sum_{i}^{\infty} \mathbf{G}$
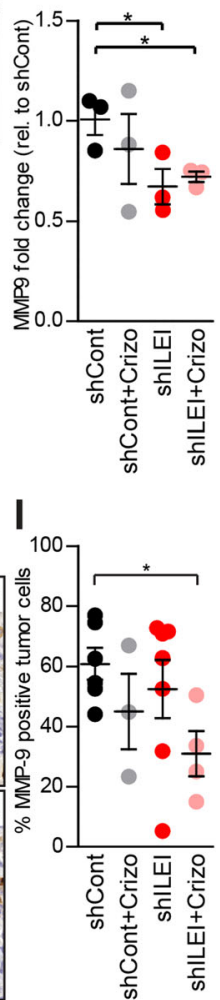

J

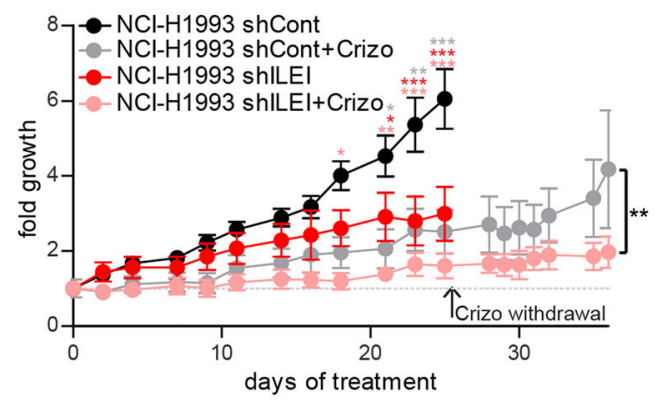

K

L

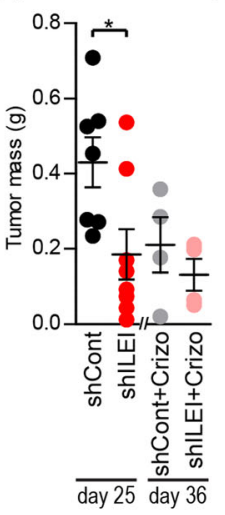

M

$\mathbf{N}$

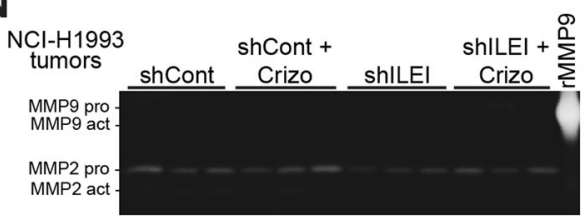

0
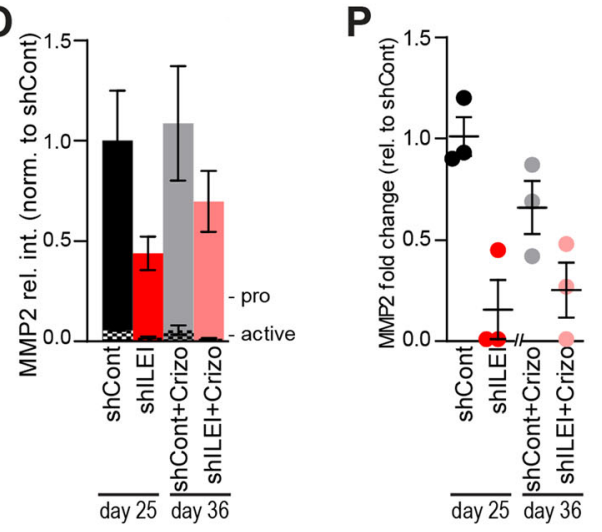

Fig. 6 (See legend on next page.) 


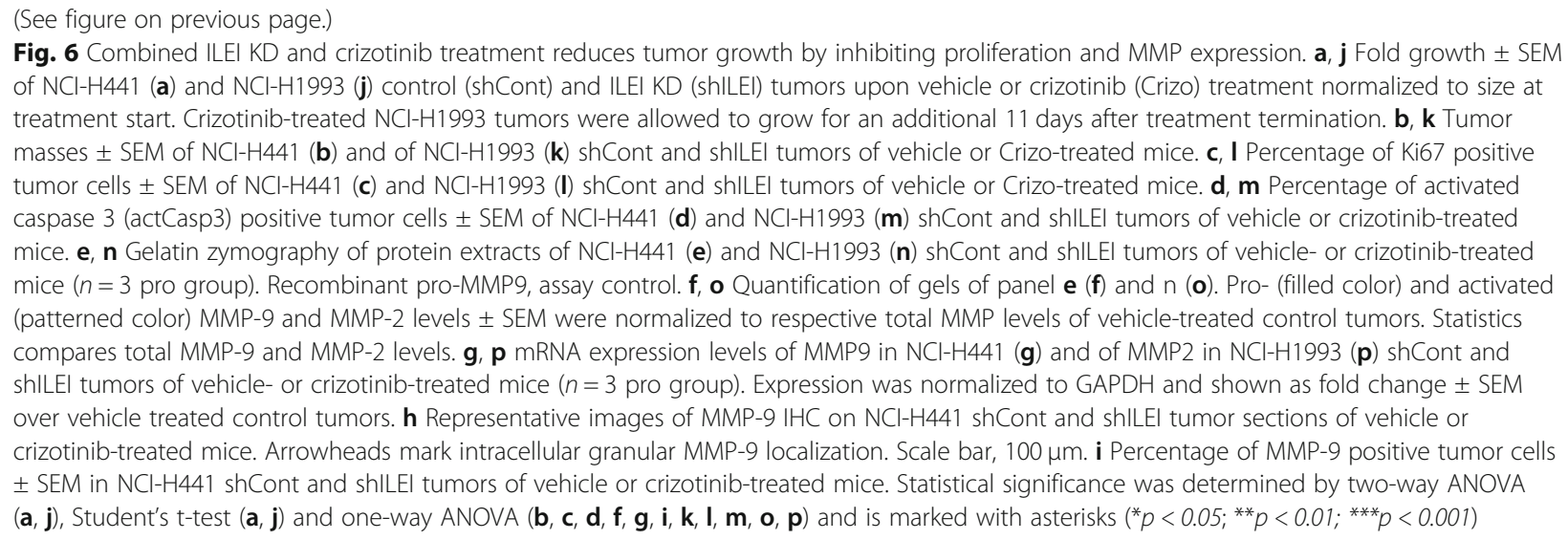

counteract the resistance of NCI-H441 cells most probably via non-cell intrinsic mechanisms not addressed here. Importantly for this study, ILEI KD also slowed the growth of both xenografts. Tumor growth was most efficiently reduced when ILEI KD was combined with crizotinib (Fig. 6a, j). The tumor mass was significantly decreased in cells with ILEI KD compared to those with ILEI expression and was lowest in cells with ILEI KD and crizotinib combined (Fig. 6b, k). Immunohistochemistry for ILEI (Supplemental Fig. S4a, g), c-MET (Supplemental Fig. S4b, h), and phospho-cMET (Supplemental Fig. S4c, i) confirmed significant ILEI KD, unaltered cMET expression upon ILEI KD and inhibitor treatment, as well as efficient inhibition of c-MET activation by crizotinib, respectively. The latter was also quantified as percentage of phospho-c-MET positive tumor cells (Supplemental Fig. S4d, j). It is notable that decrease in cMET activation was no more evident in the NCI-H1993 derived tumors due to crizotinib withdrawal over the last 11 days of the experiment (Supplemental Fig. S4j).

To investigate the reasons for smaller tumors resulting from crizotinib treatment and ILEI KD we investigated the proliferation and apoptosis of the tumor cells by quantifying the percentage of Ki67 and activated Caspase3 positive cells on tissue sections, respectively. In NCI-H441 xenografts, crizotinib treatment significantly reduced the proliferation of cells, but ILEI KD alone did not influence this parameter (Fig. 6c) [26]. So, this result supported the cell-based assays and showed that the cells were behaving in a similar manner in vivo to the in vitro analysis. In NCI-H1993 xenografts, the graph shows the recovery of the tumor cells after crizotinib treatment was halted on day 25 and observed for an additional 11 days. This indicated that after a period of drug withdrawal, proliferation was apparently no longer affected by the previous crizotinib treatment (Fig. 6l). Apoptosis was also decreased in NCI-H441 xenografts treated with crizotinib and in ILEI KD derived cells (Fig. 6d). In NCI-H1993 xenografts that had a withdrawal period from the crizotinib treatment phase there was no apparent significant difference in apoptosis with crizotinib or ILEI KD (Fig. $6 \mathrm{~m}$ ). So, the smaller tumors are likely to be due to the decreased proliferation upon crizotinib treatment rather than an increase in apoptotic cell death. A higher level of apoptosis in larger tumors without c-MET inhibition or ILEI KD may be indicative of the fast turnover of cells in these rapidly proliferating tumors that was also manifested in a highly ulcerated appearance.

We also addressed if c-MET and ILEI had a consequence on tumor vascularization by determining blood vessel density and size on CD31 immunostained tumor sections. The vessel density remained constant between tumors (Supplemental Fig. S4e, k), and though NCIH441 tumors showed decreased vessel size upon crizotinib treatment, it was less evident in the tumors with ILEI KD and not evident in any of the NCI-H1993 tumors (Supplemental Fig. S4f, 1). These data indicate that decreased tumor size upon combined ILEI KD and cMET inhibition is not primarily due to a switch in vascularization capabilities.

\section{Combined ILEI KD and crizotinib treatment decreased MMP expression in $\mathrm{NCl}-\mathrm{H} 441$ and $\mathrm{NCl}-\mathrm{H} 1993$ tumor xenografts}

To investigate whether the relationship between MMPs, c-MET, and ILEI seen in the cell lines was also evident in vivo, the expression of MMP-9 and MMP-2 from the tumors was investigated. In line with the results from gelatin zymography, the expression of MMP-9 protein in NCI-H441 tumors decreased slightly with crizotinib inhibition and even more when the cells also had ILEI KD, while MMP-2 expression, that became detectable only at in vivo conditions, was significantly decreased in ILEI KD tumors (Fig. 6e, f). At mRNA level, there was a significant decrease of MMP-9 in tumors from ILEI KD cells (Fig. 6g) and quantification of MMP-9 immunohistochemistry in tumor sections showed a similar trend 
with a significant difference between the shCont tumors and those with ILEI KD and crizotinib in combination (Fig. 6h, i). NCI-H1993 tumors also expressed lower levels of MMP-2 protein upon ILEI KD (Fig. 6n, o) and this result was supported at the mRNA level, though without significance (Fig. 6p). Overall, these results show that both c-MET and ILEI cooperate for efficient MMP expression during growth of tumor xenografts and support the results from the cell-based assays.

\section{Combined ILEI KD and crizotinib treatment increased E- cadherin membrane localization}

To further compare tumor invasiveness and EMT status, E-cadherin-mediated cell-cell adhesion was investigated. In both, NCI-H441 and NCI-H1993 xenografts immunohistochemistry of tumor sections showed a slight increase of E-cadherin at the membranes of tumors treated with crizotinib and those derived from ILEI KD cells, and this became significant when they were in combination (Fig. 7a, b, f, g). Similar to their in vitro behavior, none of the two xenografts showed regulation of E-cadherin expression at the mRNA level upon different conditions (Fig. 7c, h), nor E-cadherin levels of tumor protein extracts showed a uniform trend of regulation (Fig. 7d, e, i, j). Therefore, these results suggest that ILEI and c-MET mainly cooperate to reduce E-cadherin protein localization at the membrane to decrease cell-cell adhesion and increase the potential for invasion.

\section{Discussion}

The aim of this study was to investigate whether the FAM $3 C \mathrm{CN}$ contributes to elevated ILEI expression in cancer and its potential relationship to $M E T$. The results show a close correlation between FAM $C$ and $M E T$ CNs and that cancers with high $\mathrm{CN}$ had higher gene expression levels of both ILEI and c-MET, which was also related to poorer outcome. Investigation of the mechanisms involved suggests that there is a cooperation between ILEI and c-MET signaling during cancer invasion as summarized in the model in Fig. 8a. During c-MET-dependent invasion, as seen in some previous studies, MMP secretion was increased [23, 24] and Ecadherin levels at the cell membranes were decreased $[27,28]$. This study showed that both these processes were supported by ILEI expression and that c-MET also increased the secretion of ILEI. The secretion of active ILEI requires mobilizing its intracellular protein pool in a urokinase-type plasminogen activator receptor (uPAR)-dependent manner [13]. So, we suggest that i) cMET might be involved in that process and ii) regulatory functions of c-Met on invasion might work indirectly via regulating ILEI secretion and thus, ILEI signaling activity. c-MET signaling was not cross-regulated by ILEI and ILEI did not have an influence on c-MET- dependent proliferation in cancer cell lines, showing that the interplay between these two signaling pathways on proliferation, invasion, and overall tumor growth acts rather in a complimentary manner as shown in Fig. 8b.

Although the $M E T$ gene undergoes many different types of mutation to become oncogenic, amplification of the $M E T$ locus has been reported in a variety of human cancers [4]. Amplified $M E T \mathrm{CN}$ has been shown to negatively influence patient survival in a lot of cancer types including esophageal squamous cell carcinoma [29], NSCLC [30], clear-cell renal cell carcinoma [31], and ovarian carcinoma [32]. However, this association with poor prognosis is not always evident with high cMET protein expression [32, 33], highlighting the complexities of the $M E T$ amplicon's involvement in cancer. This observation also suggests that additional genes of the amplified region might contribute to poor patient survival. The focus of this study concentrated on one of $M E T$ 's closest neighbors, FAM3C. For its gene product, ILEI, high levels of protein expression correlated with poor prognosis in colorectal cancer [14]. We found in this study that $F A M 3 C$ frequently co-amplified with $M E T$ and patients with $M E T$ and $F A M 3 C$ amplification had poor prognosis. The high efficiency of the combined inhibition of c-MET and ILEI function on the inhibition of invasion and tumor growth of cancer cells bearing $M E T$ and FAM $3 C$ amplifications found in this study justifies the relevance of this co-amplification on clinical outcomes. Other neighboring genes in close proximity with possible co-amplification, e.g. Wnt family members and $B$-Raf, might have additional modulatory effects on c-MET and/or ILEI action and future studies of them will be interesting to fully resolve all functionally important players of this amplification hotspot in cancer.

A previous study about the variety of $M E T$ mutations in cancer used database analysis of 14,466 cancer cases and identified 186 cases with MET CN amplification [34]. This is around 1\%, similar to the TCGA database analysis in our study which showed $\mathrm{CN}$ amplification rates of $1.9 \%$ (8 in 501) for LUSC cases, $3.5 \%$ (18 in 516) for LUAD cases, $0.7 \%$ (11 of 1480) for LIHC cases, $0.2 \%$ (1 of 615$)$ for COADREAD cases, and 0.9\% (10 in 1080) for BRCA cases, with a total rate of $1.1 \%$ (48 in 4192). For further comparison, increased $M E T$ gene $\mathrm{CN}$ measured by fluorescence in situ hybridization was found in 1 to $4 \%$ of tumors from NSCLCs [30], 8.5\% of lung sarcomatoid carcinomas [35], and $4.2 \%$ of colorectal cancer tissue samples [36]. However, these values are lower than the $47 \%$ rate in cancer cell lines and $67 \%$ in colon carcinoma patient samples that had $\mathrm{CN}$ amplification of $M E T$ and FAM3C. The main reason for the differences observed between database, cell lines and patient samples are different cut-off points. We considered a $\mathrm{CN}$ of 3 or higher to be amplification in $\mathrm{qPCR}$ and gene chip 


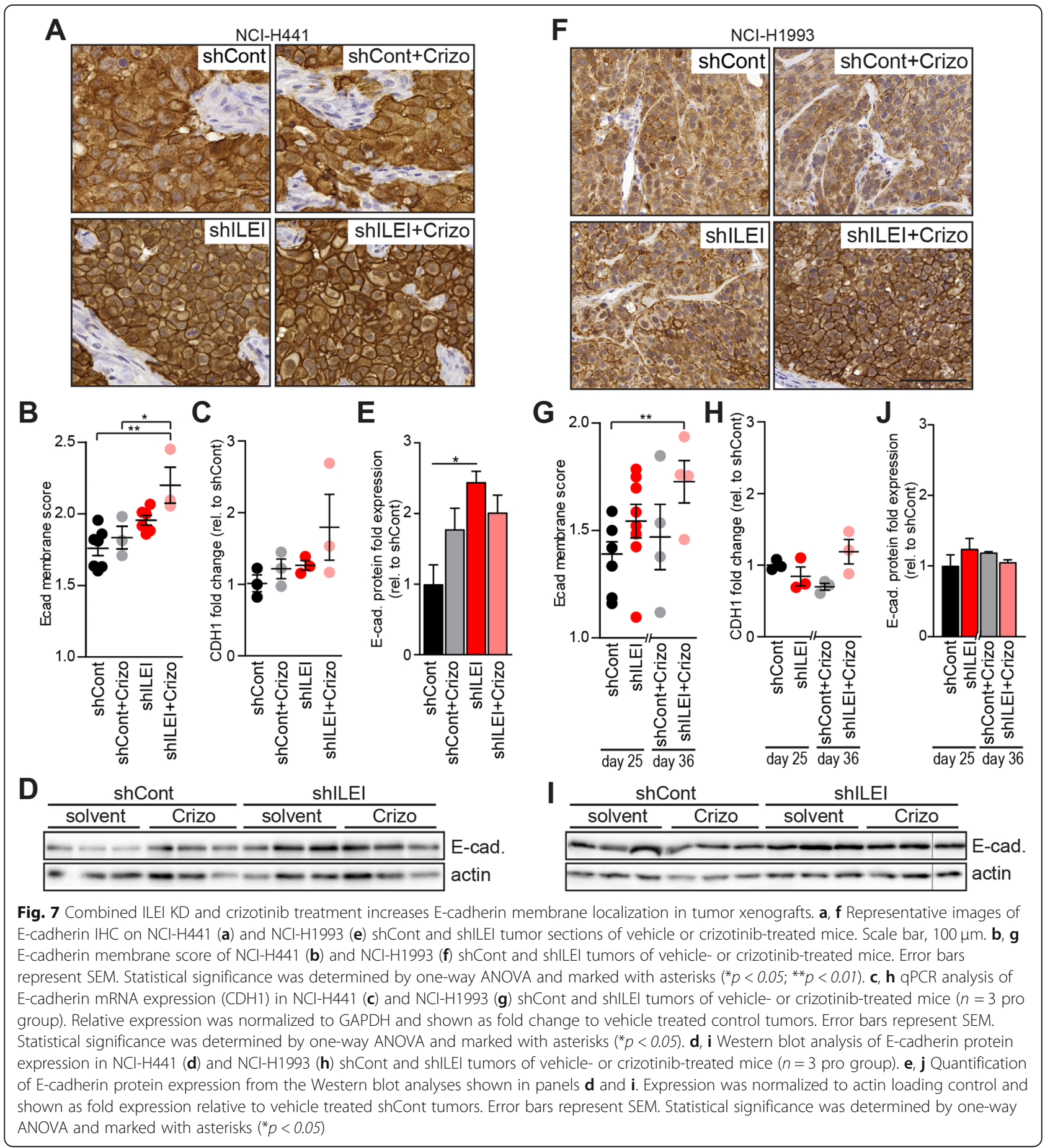

analyses of this study, while databases use higher cut-off points and a $\mathrm{CN}$ of 3 would be recorded as a gain rather than amplification. When the higher numbers of cases with gains in $\mathrm{CN}$ were added to the amplified $\mathrm{CNs}$ for the TCGA analysis the overall rate of $\mathrm{CN}$ amplification increased to nearly $30 \%$ of the total cases, which is much closer to the rates for the cell lines and COAD patients. The differences in $\mathrm{CN}$ frequencies that remain between the different samples might result from various factors. As c-MET activation leads to increased proliferation and growth of cancer cells, it is possible that this type of growth factor receptor gene amplification will be of benefit during selection of stable cancer cell lines. Indicating that, cancer cell lines might be more likely to gain $\mathrm{CN}$ amplifications. Furthermore, the 49 colorectal carcinoma cases in this study were all advanced stage 
A

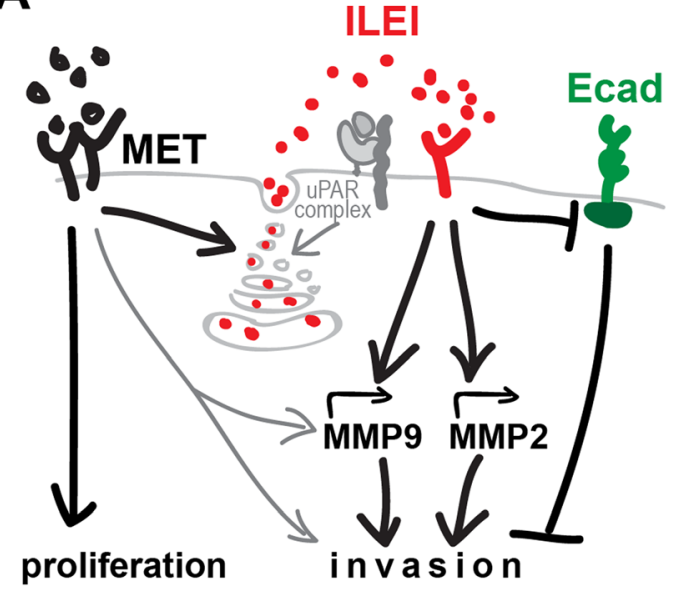

B

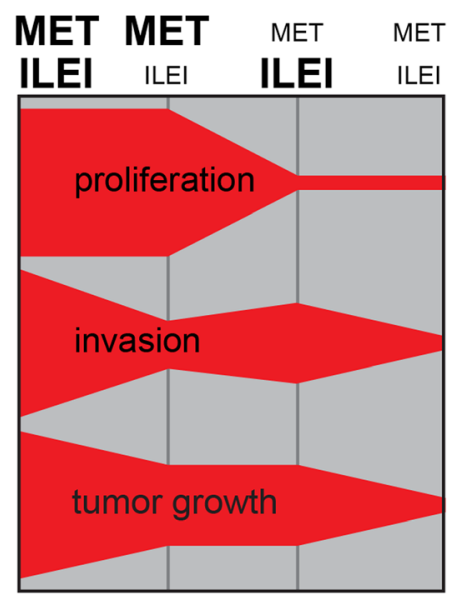

Fig. 8 Model of ILEI and c-MET signaling interplay on invasive tumor growth. a Molecular interplay of ILEI and c-MET signaling on invasion. b The influence of c-MET and ILEI signaling levels on the net outcome of tumor proliferation, invasion and overall tumor growth

cancer patients at stage T3 and T4, with 55\% already displaying lymph node and/or distant metastasis, which as our data suggests have invasion and poorer prognosis, that might result an additional enrichment of $M E T$ and $F A M 3 C \mathrm{CN}$. Our results are also in agreement with the conclusion of the above mentioned database analysis that MET CNs in general show a wide variation among cancer types, albeit relative frequencies for different tumor entities show differencies between the studies.

All these data show that $M E T$ amplification has broad relevance in many cancers and our study indicates that FAM $3 C$ co-amplification may play a comparably important role in all these different cancer types. As the alternative name of c-MET indicates, Hepatocyte Growth Factor Receptor (HGFR) has an important role in liver development and regeneration [37], thus predestinating a pivotal oncogenic driver function of the gene in liver cancer. Indeed, aberrant c-MET function, including gene amplification is frequent in HCC [38]. However, the above statistics show that it is not less frequent in other epithelial cancer types, and MET amplification is one of the frequent aquired alterations (5-20\% of patients) upon resistance towards EGFR tyrosine kinase inhibitor (TKI) therapies in lung cancer [39]. Thus, our mechanistic studies on NSCLC cell lines with MET amplification are of direct clinical relevance to many cancer types.

c-MET signaling involves many different processes and significant crosstalks with other signaling pathways. For example, there is an interaction between c-MET signaling and the vascular endothelial growth factor (VEGF) and VEGF receptor (VEGFR) pathways [40]. While interactions between c-MET and human epidermal growth factor receptor (HER) family members allow tumor progression and treatment resistance, and cooperative signaling between c-MET and HER2 might be a mechanism by which c-MET promotes cancer progression [41]. Interestingly, in breast cancer cells the ILEIUPAR score, which is indicative of the potential for active secreted ILEI, was shown to be significantly correlated with the HER2 status of the tumor cells [13] suggesting that these three signaling pathways may cooperate to increase invasiveness. In addition, uPARbound activated UPA is required for the proteolytic maturation of the c-MET ligand HGF [4]. At the same time, active $\mathrm{UPA}$ is needed for the activation of Plasminogen, the protease responsible for the maturation of ILEI, and activation of the UPA-uPAR system has also a key role in triggering ILEI secretion [13]. This shared use of the same proteolytic cascade by both signaling pathways for activation also indicates a strong positive regulatory connection. Because of the importance of c-MET in many cancer types, inhibitors of c-MET are in clinical trials as cancer treatment, but a significant percentage of tumors acquire resistance to these treatments $[4,19]$. This may in part be due to the ability of c-MET to crosstalk and interact with alternative RTKs such as VEGFR and HER2 [40, 41]. Combination treatments may help address this problem and the results of this study suggest that ILEI might be a potential target for these treatments as highlighted by the xenograft experiments that showed tumor growth was mostly inhibited by combined crizotinib and ILEI KD.

ILEI is less well understood than c-MET, but its role in cancer progression is starting to emerge. ILEI translation appears to be stimulated by transforming growth factor beta (TGF- $\beta$ ) and silenced by heterogeneous nuclear ribonucleoprotein E1 (hnRNP E1). Part of the signaling pathway, at least in breast cancer cells, involves the leukemia inhibitory factor receptor (LIFR) and signal transducer and activator of transcription 3 (STAT3) 
signaling [42]. The active form of ILEI requires proteolysis and is self-dimerized [13, 17, 43]. However, it was unclear so far whether over-expression of ILEI could result from $\mathrm{CN}$ amplification until this study.

Once we had established that $M E T$ and FAM3C were often co-amplified and this led to combined overexpression of c-MET and ILEI, it was important to investigate whether they interact during cancer progression. For this, we used five cancer cell lines that had shown coamplified $M E T$ and $F A M 3 C$. For c-MET inhibition, we used crizotinib and ILEI KD was used to mimic ILEI inhibition. Somewhat different to the published data on the MET inhibitor PHA665752 [19], OE33 cells showed high sensitivity to increasing concentrations of crizotinib in proliferation capacity and were also sensitive to both PHA665752 and savolitinib in this study. The other cell lines showed in vitro sensitivity (NCI-H1993 and MKN45) or resistance (NCI-H441 and SKBR3) towards crizotinib as expected from previous publication. ILEI KD did not influence the proliferation of the cell lines suggesting that ILEI is not involved in proliferation of cancer cells or c-MET-regulated proliferation. However, ILEI KD strongly impaired the invasiveness of all five cancer cell lines. This was in accordance with earlier findings [6] and further supported that view that ILEI signaling induces invasiveness in different types of cancer [44]. Importantly, the impairment of invasiveness by ILEI KD on HGF-induced, hence c-MET-dependent invasion suggests that c-MET driven invasion depends on ILEI. In addition, the importance of ILEI in c-METindependent invasion suggests that ILEI has a broad influence on invasion and does not rely exclusively on cMET secretion trigger.

In terms of invasion, an important step is ECM degradation which allows tumor dissemination. MMPs are implicated in this process because they mediate the constant remodeling of the ECM [22]. Our results suggested that ILEI was required for HGF-induced expression and secretion of MMP-9 and MMP-2 through c-MET signaling. Although the expression levels of ILEI were not influenced by crizotinib treatment and ILEI did not affect activation of c-MET signaling, there was an apparent decrease in ILEI secretion upon c-MET inhibition. This suggests c-MET may regulate MMPs indirectly via ILEI secretion. So, the independent c-MET and ILEI processes show a vital interplay and cooperation to support invasiveness.

The results of the cell-based studies were then further investigated in xenografts in mice. Our expectation was that the growth of crizotinib-sensitive tumors would be slower than for crizotinib resistant tumors when c-MET was inbitited. However, crizotinib reduced the growth and tumor mass of xenografts induced by both sensitive (NCI-H1993) and resistant (NCI-H441) cell types. This was is in accordance with some earlier findings [26] and suggested that crizotinib was able to counteract the resistence of NCI-H441 cells to inhibit c-MET, most probably via non-cell intrinsic mechanisms that will require further investigation. This result meant that we then focused on ILEI and found that both cell types showed reduced growth of tumor xenografts from ILEI KD and a superior reduction with crizotinib treatment in combination supporting the interplay between the two signaling pathways. Combination of ILEI KD and crizotinib also resulted in lower expression of MMPs in a similar way to the cell-based studies. MMP-2 and MMP-9 can degrade components of the ECM such as type IV collagen to release tension and allow growth and invasion of tumors and as such are implicated in the late stages of cancer [45]. During EMT cell-cell junctions begin to disassemble [9]. The best characterized alteration at this point involves the loss of E-cadherin, a key cellto-cell adhesion molecule [25]. E-cadherin helps to assemble and maintain epithelial cell sheets through adherence junctions. Therefore, increased expression of E-cadherin acts as an antagonist of invasion and metastasis [46]. When E-cadherin was investigated in the xenograft tumors, combined crizotinib and ILEI KD significantly increased E-cadherin at the membranes. This was less obvious at the mRNA and protein levels. Overall, our study showed high variance among tumor cells how E-cadherin levels were regulated, indicating that it is a highly dynamic process with strong control at the transcription level, but also via internalization or altered stability in cancer cells [47]. Also, in some cancers, such as hepatocellular carcinoma, E-cadherin protein accumulation is prevented by mRNA retention in the nucleus [48].

\section{Conclusions}

The results of this study show that amplification of FAM3C $\mathrm{CN}$ can contribute to increase the level of ILEI expression in a wide range of cancer types. There was a close correlation between $F A M 3 C$ and $M E T$ CNs in cancer patients and those with high $\mathrm{CNs}$ had poorer outcomes. Investigation of the mechanisms involved showed interplay between the two separate ILEI and c-MET signaling pathways during cancer invasion, suggesting $M E T$ amplifications are in reality $M E T-F A M 3 C$ co-amplifications with tight functional co-operation. In vivo investigation showed that ILEI knock-down and c-MET-inhibition in combination significantly reduced the invasive outgrowth of lung tumor xenografts in mice, apparently by inhibiting proliferation, MMP expression and E-cadherin membrane localization. Therefore, including ILEI as a 
target in the therapy of cMET-amplified human carcinomas may be an effective approach for the future.

\section{Supplementary Information}

The online version contains supplementary material available at https://doi. org/10.1186/s13046-021-01862-5.

\section{Additional file 1: Figure S1. (related to Fig. 1.). FAM3C and MET gene} copy numbers are tightly linked in human hepatocellular, colorectal and breast cancers with FAM $3 C$ amplification correlating with venous invasion in colorectal cancer patients. a Summary of MET, FAM3C, EGFR and FGFR1 copy number calls extracted from hepatocellular carcinoma LIHC (left panel), colorectal adenocarcinoma COADREAD (mid panel) and breast carcinoma BRCA (right panel) TCGA datasets. Genomic localization is marked above the genes. GISTIC 2.0 values are shown: -2 , deep deletion (DD); - 1, shallow deletion (SD); 0, diploid (Dipl); 1, gain; 2, amplification (Amp). Numbers within the boxes refer to the number of tumors within a group. Orange connection lines indicate tumor samples with FAM3C gain or amplification. Correlation was calculated using Kendall's tau-b test. b Heatmap with hierarchical clustering of FAM $3 C$ and MET gene amplification determined by Taqman $\mathrm{QPCR}$ and of extramural venous invasion $(E M V I)$, tumor stage $(\mathrm{pT})$, lymph node status $(\mathrm{pN})$ and metastasis status (pM) clinicopathological parameters extracted from 49 primary colon carcinoma patients. Calculated copy numbers of 3 or higher were considered as gene amplification. Correlation was calculated using Kendall's tau-b test. c Correlation of FAM3C gene amplification status with established clinicopathological parameters of the 49 human colorectal cancer patients shown in panel b. The number of patients (n) and their relative frequencies (in \%; numbers in parentheses) in the indicated clinicophathological categories are shown. $p$-value, Chi-square test $p$-values.

Additional file 2: Figure S2. (related to Fig. 3.). The five selected cancer cell lines and their ILEI KD derivatives show similar sensitivity towards the c-MET inhibitors PHA665752 and savolitinib as for crizotinib. a, b Viability of NCl-H1993, NCl-H441, MKN45, OE33 and SKBR3 cells towards increasing concentrations of the c-MET inhibitors PHA665752 (a) and savolitinib (b). Fitting curve was normalized to untreated control condition. Dashed line marks IC50.

Additional file 3: Figure S3. (related to Fig. 5.). The selective c-MET inhibitor savolitinib reproduces the effects of crizotinib on ILEl secretion, HGF-induced expression of MMPs and E-cadherin expression. a Western blot analysis of ILEl secretion and expression, and c-MET activity and expression in the five selected cell lines (parental) and their control (shCont) and ILEI KD (sh261 and sh506) derivatives after savolitinib $(1 \mu \mathrm{M})$ treatment for $24 \mathrm{~h}$. b aPCR analysis of MMP-9 (for NCl-H441, MKN45 and OE33) and MMP-2 (for NCl-H1993 and SKBR3) mRNA expression in control (shCont) and ILEI KD (shlLEI) cells after $24 \mathrm{~h}$ of HGF treatment $(40 \mathrm{ng} / \mathrm{ml})$ in the absence or presence of savolitinib $(1 \mu \mathrm{M})$. Data are normalized as fold change to untreated control cells. Error bars represent SEM of three independent experiments. Statistical significance was determined by oneway ANOVA. c $\mathrm{QPCR}$ analysis of E-cadherin mRNA expression (CDH1) in NCI-H1993, NCl-H441, MKN45, OE33 and SKBR3 control (shCont) and ILEI KD (shILEI) cells treated or non-treated with savolitinib $(1 \mu \mathrm{M})$ for $24 \mathrm{~h}$. Data are normalized as fold change to untreated control cells. Error bars represent SEM of three independent experiments. Statistical significance was determined by one-way ANOVA and marked with asterisks $\left({ }^{*} p<0.05\right.$; ${ }^{* *} p<0.01 ;{ }^{* * *} p<0.001, p<0.001 ;{ }^{* * *} p<0.0001$ ).

Additional file 4: Figure S4. (related to Fig. 6.). The effect of ILEI KD and crizotinib treatment on ILEl expression, c-Met expression and phosphorylation and tumor vessel formation. $\mathbf{a}, \mathbf{g}$ Representative images of ILEI IHC on NCl-H441 (a) and NCl-H1993 (g) shCont and shlLEl tumor sections of vehicle or Crizo treated mice. Scale bar, $100 \mu \mathrm{m}$. b, h Representative images of CMET IHC on NCl-H441 (b) and NCl-H1993 (h) shCont and shILEl tumor sections of vehicle or Crizo treated mice. Scale bar, $100 \mu \mathrm{m}$. c, i Representative images of phospho-CMET IHC on NCI-H441 (c) and $\mathrm{NCl}-\mathrm{H} 1993$ (i) shCont and shlLEl tumor sections of vehicle or Crizo treated mice. Scale bar, $100 \mu \mathrm{m}$. d, $\mathbf{j}$ Quantification of cMET activation in NClH441 (d) and NCl-H1993 (j) shCont and shlLEl tumors of vehicle or Crizo treated mice determined as percentage of phospho-cMET (PMET) positive tumor cells on IHC stained tissue sections. Note, in NCl-H1993 xenografts crizotinib was withdrawn 11 days before time point of analysis. Error bars represent SEM. Statistical significance was determined by one-way ANOVA. e, f, k, I Quantification of tumor vessels in NCl-H441 (e, f) and $\mathrm{NCl}-\mathrm{H} 1993(k, 1)$ shCont and shILEl tumors of vehicle or Crizo treated mice by average vessel density $(e, k)$ and average vessel size $(f, l)$ as determined from CD31 stained tumor tissue sections.

\section{Abbreviations}

ANOVA: Analysis of variance; CM: Conditioned medium; CN: Copy number; ECL: Enhanced chemiluminescent; DAB: 3,3'-diaminobenzidine; DAPI: 4',6diamidino-2-phenylindole; EGFR: Epidermal growth factor receptor; EMT: Epithelial-mesenchymal transition; EMVI: Extramural venous invasion; FCS: Fetal calf serum; FGFR1: Fibroblast growth factor receptor 1; HER: Human epidermal growth factor receptor; HGF: Hepatocyte growth factor; H\&E: Hematoxylin and eosin; hnRNP E1: Heterogeneous nuclear ribonucleoprotein E1; ILEl: Interleukin-like EMT inducer; KD: Knock-down; LIFR: Leukemia inhibitory factor receptor; LUAD: Lung adenocarcinoma; LUSC: Lung squamous cell carcinoma; MMP: Matrix metalloproteinase; MTT: 3-(4,5-dimethylthiazol-2-yl)-2,5-diphenyltetrazolium bromide; NSCL C: Non-small-cell lung carcinoma; PBS: Phosphate-buffered saline; SCID: Severe combined immunodeficiency disease; SEM: Standard error of the mean; STAT3: Signal transducer and activator of transcription 3; TGF$\beta$ : Transforming growth factor beta; UPAR: Urokinase-type plasminogen activator receptor; VEGF: Vascular endothelial growth factor; VEGFR: VEGF receptor

\section{Acknowledgements}

We thank Danijela Kurija, Mia Gössinger and Gerhard Zeitler for technical assistance. We thank Wolfgang Mikulits for providing sequence information of validated QPCR primers for genes analysed in this study and Gergely Szakacs for fruitful discussions and comments on the manuscript. Writing assistance was also provided by Melanie Colegrave of Molecular Cell Research.

\section{Authors' contributions}

US had major contribution in designing and performing experiments including most work related to generation of stable ILEI KD cell lines and in vitro functional assays; GH performed data extraction and computational analysis from the TCGA database; GT contributed in microscopic imaging, computational data analysis and manuscript editing; $\mathrm{PH}$ performed the in vivo mouse xenograft experiments and edited the manuscript; NS contributed with material and data sharing and computational data analysis; ZS provided primary human material and performed data extraction and analysis. MS provided advice in experimental design, took up mentorship and edited the manuscript; WB provided advice in experimental design, coordinated in vivo mouse xenograft experiments and edited the manuscript; AC developed the hypothesis, designed, performed and financed experiments, wrote the manuscript and coordinated the study. All authors contributed to draft corresponding parts of the manuscript and read and approved the final manuscript.

\section{Funding}

This work was supported by the Stand-alone Project P25834-B23 (AC) of the Austrian Science Foundation FWF.

\section{Availability of data and materials}

GISTIC 2.0 copy number calls were extracted from the TCGA datasets BRCA, HNSC, LUAD and LUSC using the download function of cBio database (http://www.cbioportal.org/). Other datasets generated and/or analyzed during the current study are available from the corresponding author on reasonable request.

\section{Ethics approval and consent to participate}

De-identified advanced stage colorectal tissues were obtained from stored samples from patients treated in Kecskemet General Hospital, Hungary, who had previously provided informed consent for their use in clinical research. All animal work was done by following earlier protocols ethically approved by the Institutional Animal Care and Use Committee of the Medical 
University of Vienna and by the Austrian Bundesministerium für Bildung, Wissenschaft und Forschung (BMWFW-66.009/0081-WF/N/3b/2015).

\section{Consent for publication}

Not applicable.

\section{Competing interests}

The authors declare that they have no competing interests.

\section{Author details}

${ }^{1}$ Research Institute of Molecular Pathology, Dr. Bohr-Gasse 3, A-1030 Vienna, Austria. ${ }^{2}$ Department of Medicine I, Division of Oncology, Medical University of Vienna, Währinger Gürtel 18-20, A-1090 Vienna, Austria. ${ }^{3}$ Department of Medicine I, Institute of Cancer Research, Medical University of Vienna, Borschkegasse 8a, A-1090 Vienna, Austria. ${ }^{4}$ Department of Oncology, Bacs-Kiskun County Teaching Hospital, Kecskemet, Hungary. ${ }^{5}$ Present Address: Parexel International, 2 Federal St, Billerica, MA, USA.

${ }^{6}$ Boehringer-Ingelheim RCV GmbH \& Co KG, Vienna, Austria.

\section{Received: 27 October 2020 Accepted: 1 February 2021}

Published online: 17 February 2021

\section{References}

1. Hanahan D, Weinberg RA. Hallmarks of cancer: the next generation. Cell. 2011;144(5):646-74.

2. Hnisz D, Weintraub AS, Day DS, Valton AL, Bak RO, Li CH, et al. Activation of proto-oncogenes by disruption of chromosome neighborhoods. Science. 2016;351(6280):1454-8.

3. Organ SL, Tsao MS. An overview of the c-MET signaling pathway. Ther Adv Med Oncol. 2011:3(1 Suppl):S7-S19.

4. Blumenschein GR Jr, Mills GB, Gonzalez-Angulo AM. Targeting the hepatocyte growth factor-cMET axis in cancer therapy. J Clin Oncol. 2012; 30(26):3287-96.

5. Hyman E, Kauraniemi P, Hautaniemi S, Wolf M, Mousses S, Rozenblum E, et al. Impact of DNA amplification on gene expression patterns in breast cancer. Cancer Res. 2002:62(21):6240-5.

6. Waerner T, Alacakaptan M, Tamir I, Oberauer R, Gal A, Brabletz T, et al. ILEl: a cytokine essential for EMT, tumor formation, and late events in metastasis in epithelial cells. Cancer Cell. 2006;10(3):227-39.

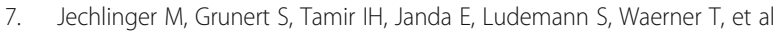
Expression profiling of epithelial plasticity in tumor progression. Oncogene. 2003;22(46):7155-69.

8. Du B, Shim JS. Targeting epithelial-Mesenchymal transition (EMT) to overcome drug resistance in cancer. Molecules. 2016;21:7.

9. Micalizzi DS, Farabaugh SM, Ford HL. Epithelial-mesenchymal transition in cancer: parallels between normal development and tumor progression. J Mammary Gland Biol Neoplasia. 2010;15(2):117-34.

10. Liao T, Yang MH. Revisiting epithelial-mesenchymal transition in cancer metastasis: the connection between epithelial plasticity and stemness. Mol Oncol. 2017:11(7):792-804.

11. Lahsnig C, Mikula M, Petz M, Zulehner G, Schneller D, van Zijl F, et al. ILEl requires oncogenic Ras for the epithelial to mesenchymal transition of hepatocytes and liver carcinoma progression. Oncogene. 2009;28(5):638-50.

12. Song $Q$, Sheng W, Zhang X, Jiao S, Li F. ILEl drives epithelial to mesenchymal transition and metastatic progression in the lung cancer cell line A549. Tumour Biol. 2014;35(2):1377-82.

13. Csiszar A, Kutay B, Wirth S, Schmidt U, Macho-Maschler S, Schreiber M, et al. Interleukin-like epithelial-to-mesenchymal transition inducer activity is controlled by proteolytic processing and plasminogen inverted question mark urokinase plasminogen activator receptor system-regulated secretion during breast cancer progression. Breast Cancer Res. 2014;16(5):433.

14. Gao ZH, Lu C, Wang ZN, Song YX, Zhu JL, Gao P, et al. ILEl: a novel marker for epithelial-mesenchymal transition and poor prognosis in colorectal cancer. Histopathology. 2014;65(4):527-38.

15. Weksberg R, Hughes S, Moldovan L, Bassett AS, Chow EW, Squire JA. A method for accurate detection of genomic microdeletions using real-time quantitative PCR. BMC Genomics. 2005;6:180

16. Smolen GA, Sordella R, Muir B, Mohapatra G, Barmettler A, Archibald H, et al. Amplification of MET may identify a subset of cancers with extreme sensitivity to the selective tyrosine kinase inhibitor PHA-665752. Proc Natl Acad Sci U S A. 2006;103(7):2316-21.
17. Kral M, Klimek C, Kutay B, Timelthaler G, Lendl T, Neuditschko B, et al. Covalent dimerization of interleukin-like epithelial-to-mesenchymal transition (EMT) inducer (ILEI) facilitates EMT, invasion, and late aspects of metastasis. FEBS J. 2017:284(20):3484-505.

18. Streit M, Lex A, Kalkusch M, Zatloukal K, Schmalstieg D. Caleydo: connecting pathways and gene expression. Bioinformatics. 2009;25(20):2760-1.

19. McDermott U, Sharma SV, Dowell L, Greninger P, Montagut C, Lamb J, et al. Identification of genotype-correlated sensitivity to selective kinase inhibitors by using high-throughput tumor cell line profiling. Proc Natl Acad Sci U S A. 2007;104(50):19936-41.

20. Ikebuchi F, Oka K, Mizuno S, Fukuta K, Hayata D, Ohnishi H, et al. Dissociation of c-met phosphotyrosine sites in human cells in response to mouse hepatocyte growth factor but not human hepatocyte growth factor: the possible roles of different amino acids in different species. Cell Biochem Funct. 2013;31(4):298-304.

21. Rong S, Bodescot M, Blair D, Dunn J, Nakamura T, Mizuno K, et al. Tumorigenicity of the met proto-oncogene and the gene for hepatocyte growth factor. Mol Cell Biol. 1992;12(11):5152-8.

22. Winer A, Adams S, Mignatti P. Matrix metalloproteinase inhibitors in cancer therapy: turning past failures into future successes. Mol Cancer Ther. 2018; 17(6):1147-55

23. Kermorgant S, Aparicio T, Dessirier V, Lewin MJ, Lehy T. Hepatocyte growth factor induces colonic cancer cell invasiveness via enhanced motility and protease overproduction. Evidence for PI3 kinase and PKC involvement. Carcinogenesis. 2001;22(7):1035-42.

24. Fujiuchi Y, Nagakawa O, Murakami K, Fuse H, Saiki I. Effect of hepatocyte growth factor on invasion of prostate cancer cell lines. Oncol Rep. 2003; 10(4):1001-6.

25. Bure IV, Nemtsova MV, Zaletaev DV. Roles of E-cadherin and noncoding RNAs in the epithelial-mesenchymal transition and progression in gastric cancer. Int J Mol Sci. 2019;20:12.

26. Zou HY, Li Q, Lee JH, Arango ME, McDonnell SR, Yamazaki S, et al. An orally available small-molecule inhibitor of c-met, PF-2341066, exhibits cytoreductive antitumor efficacy through antiproliferative and antiangiogenic mechanisms. Cancer Res. 2007;67(9):4408-17.

27. Miura H, Nishimura K, Tsujimura A, Matsumiya K, Matsumoto K, Nakamura T, et al. Effects of hepatocyte growth factor on E-cadherin-mediated cell-cell adhesion in DU145 prostate cancer cells. Urology. 2001;58(6):1064-9.

28. Menakongka A, Suthiphongchai T. Involvement of PI3K and ERK1/2 pathways in hepatocyte growth factor-induced cholangiocarcinoma cell invasion. World J Gastroenterol. 2010;16(6):713-22.

29. Wang $Y$, Jiang $Z$, Xu C, Wang H, Tan L, Su J, et al. Increased MET gene copy number negatively affects the survival of esophageal squamous cell carcinoma patients. BMC Cancer. 2019;19(1):240.

30. Cappuzzo F, Marchetti A, Skokan M, Rossi E, Gajapathy S, Felicioni L, et al. Increased MET gene copy number negatively affects survival of surgically resected non-small-cell lung cancer patients. J Clin Oncol. 2009;27(10):1667-74.

31. Macher-Goeppinger S, Keith M, Endris V, Penzel R, Tagscherer KE, Pahernik S, et al. MET expression and copy number status in clear-cell renal cell carcinoma: prognostic value and potential predictive marker. Oncotarget. 2017:8(1):1046-57.

32. Kim WY, Shim SH, Jung HY, Dong M, Kim SN, Lee SJ. The gene copy number of c-MET has a significant impact on progression-free survival in Korean patients with ovarian carcinoma. Hum Pathol. 2017;64:98-105.

33. Lee SJ, Lee J, Sohn I, Mao M, Kai W, Park CK, et al. A survey of c-MET expression and amplification in 287 patients with hepatocellular carcinoma. Anticancer Res. 2013;33(11):5179-86.

34. Sivakumar $M$, Jayakumar $M$, Seedevi $P$, Sivasankar $P$, Ravikumar $M$, Surendar $\mathrm{S}$, et al. Meta-analysis of functional expression and mutational analysis of cmet in various cancers. Curr Probl Cancer. 2019;44:100515.

35. Mignard X, Ruppert AM, Antoine M, Vasseur J, Girard N, Mazieres J, et al. C-MET overexpression as a poor predictor of MET amplifications or exon 14 mutations in lung Sarcomatoid carcinomas. J Thorac Oncol. 2018;13(12):1962-7.

36. Kwak Y, Yun S, Nam SK, Seo AN, Lee KS, Shin E, et al. Comparative analysis of the EGFR, HER2, C-MYC, and MET variations in colorectal cancer determined by three different measures: gene copy number gain, amplification status and the 2013 ASCO/CAP guideline criterion for HER2 testing of breast cancer. J Transl Med. 2017:15(1):167.

37. Nalesnik MA, Michalopoulos GK. Growth factor pathways in development and progression of hepatocellular carcinoma. Front Biosci (Scholar edition). 2012;4:1487-515. 
38. Bouattour M, Raymond E, Qin S, Cheng AL, Stammberger U, Locatelli G, et al. Recent developments of c-met as a therapeutic target in hepatocellular carcinoma. Hepatology (Baltimore, Md). 2018;67(3):1132-49.

39. Sequist LV, Waltman BA, Dias-Santagata D, Digumarthy $S$, Turke AB, Fidias $P$, et al. Genotypic and histological evolution of lung cancers acquiring resistance to EGFR inhibitors. Sci Transl Med. 2011;3(75):75ra26.

40. Aftab DT, McDonald DM. MET and VEGF: synergistic targets in castrationresistant prostate cancer. Clin Transl Oncol. 2011;13(10):703-9.

41. Lindemann K, Resau J, Nahrig J, Kort E, Leeser B, Annecke K, et al. Differential expression of c-met, its ligand HGF/SF and HER2/neu in DCIS and adjacent normal breast tissue. Histopathology. 2007:51(1):54-62.

42. Woosley AN, Dalton AC, Hussey GS, Howley BV, Mohanty BK, Grelet S, et al. TGFbeta promotes breast cancer stem cell self-renewal through an ILEI/LIFR signaling axis. Oncogene. 2019;38(20):3794-811.

43. Jansson AM, Csiszar A, Maier J, Nystrom AC, Ax E, Johansson P, et al. The interleukin-like epithelial-mesenchymal transition inducer ILEI exhibits a non-interleukin-like fold and is active as a domain-swapped dimer. J Biol Chem. 2017;292(37):15501-11.

44. Noguchi K, Dincman TA, Dalton AC, Howley BV, McCall BJ, Mohanty BK, et al. Interleukin-like EMT inducer (ILEI) promotes melanoma invasiveness and is transcriptionally up-regulated by upstream stimulatory factor-1 (USF-1). J Biol Chem. 2018:293(29):11401-14.

45. Lustosa SA, Viana Lde S, Affonso RJ Jr, Silva SR, Denadai MV, de Toledo SR, et al. Expression profiling using a CDNA array and immunohistochemistry for the extracellular matrix genes FN-1, ITGA-3, ITGB-5, MMP-2, and MMP-9 in colorectal carcinoma progression and dissemination ScientificWorldJournal. 2014;2014:102541.

46. Bruner HC, Derksen PWB. Loss of E-cadherin-dependent cell-cell adhesion and the development and progression of cancer. Cold Spring Harb Perspect Biol. 2018;10:3.

47. Wong SHM, Fang CM, Chuah LH, Leong CO, Ngai SC. E-cadherin: its dysregulation in carcinogenesis and clinical implications. Crit Rev Oncol Hematol. 2018;121:11-22.

48. Ghafoory S, Mehrabi A, Hafezi M, Cheng X, Breitkopf-Heinlein K, Hick M, et al. Nuclear accumulation of CDH1 mRNA in hepatocellular carcinoma cells. Oncogenesis. 2015;4:e152.

\section{Publisher's Note}

Springer Nature remains neutral with regard to jurisdictional claims in published maps and institutional affiliations.

Ready to submit your research? Choose BMC and benefit from:

- fast, convenient online submission

- thorough peer review by experienced researchers in your field

- rapid publication on acceptance

- support for research data, including large and complex data types

- gold Open Access which fosters wider collaboration and increased citations

- maximum visibility for your research: over $100 \mathrm{M}$ website views per year

At $\mathrm{BMC}$, research is always in progress.

Learn more biomedcentral.com/submissions 